BDX-613-1497

Distribution Category UC-38

OPTICAL SYSTEM FOR AUTOMATED

INSPECTION OF HIGHLY REFLECTIVE

SPHERICAL SURFACES

Published February 1977

Project Leader:

P. E. Klingsporn

Department 812

Project Team:

R. T. Stagner

This report was prepared as an account of work

sponsored by the United States Government. Neither

the United States nor the United States Energy

Research and Development Ad of their ontractors,

their employees. nor

subcontractors, of implied, or assumes any legal

lisbility or resporsibility for the accuracy, completenes

or usefulness of any information, apparatus, product or

process disclosed, or represents that its use would no

infringe privately owned rights.

PDO 6984778

Topical Report

Technical Communications

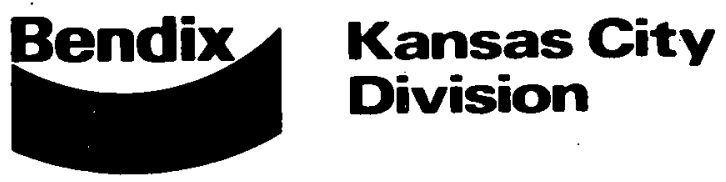

DISTRIGUTION OT THIS DOCUMANT IS UNLMMTE 


\section{DISCLAIMER}

This report was prepared as an account of work sponsored by an agency of the United States Government. Neither the United States Government nor any agency Thereof, nor any of their employees, makes any warranty, express or implied, or assumes any legal liability or responsibility for the accuracy, completeness, or usefulness of any information, apparatus, product, or process disclosed, or represents that its use would not infringe privately owned rights. Reference herein to any specific commercial product, process, or service by trade name, trademark, manufacturer, or otherwise does not necessarily constitute or imply its endorsement, recommendation, or favoring by the United States Government or any agency thereof. The views and opinions of authors expressed herein do not necessarily state or reflect those of the United States Government or any agency thereof. 


\section{DISCLAIMER}

Portions of this document may be illegible in electronic image products. Images are produced from the best available original document. 


\title{
OPTICAL SYSTEM FOR AUTOMATED INSPECTION OF HIGHLY REFLECTIVE SPHERICAL SURFACES
}

\author{
BDX-613-1497, UNCLASSIFIED Topical Report, Published February 1977
}

Prepared by P. E. Klingsporn, D/812, under PDO 6984778

An optical system was designed for use in automated inspection of superfinished spherical surfaces. Helium neon laser light is used to illuminate a small region on the spherical surface, and the light reflected from the surface is focused to a point on the face of a photodetector array capable of measuring light intensity as a function of both radial and angular coordinates. When an isolated defect such as a pit or scratch is illuminated, a characteristic diffraction pattern, centered about the focused spot, is observed in the plane of the detector array. Theoretical relations were derived which relate the intensities and sizes of the defect diffraction patterns to the type and dimensions of the isolated defect. The latter relations are important for determining defect sizes from photodetector measurements of the diffraction pattern intensities and thereby establishing surface quality certification criteria.

This report was prepared as an account of work sponsored by the United States Government. Neither the United States nor the United States Energy Research and Development Administration, nor any of their employees, nor any of their contractors, subcontractors, or their employees, makes any warranty, express or implied, or assumes any legal liability or responsibility for the accuracy, completeness or usefulness of any information, apparatus, product or process disclosed, or represents that its use would not infringe privately owned rights.
THE BENDIX CORPORATION

KANSAS CITY DIVISION

P.O. BOX 1159

KANSAS CITY, MISSOURI 64141

\footnotetext{
A prime contractor with the United States Energy Research and Development Administration Contract Number EY-76-C-04-0613 USERDA
} 


\section{CONTENTS}

Section $\quad$ Page

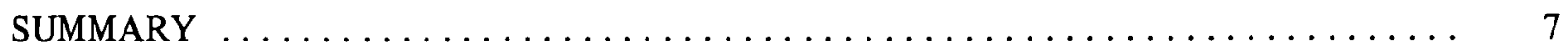

$\operatorname{DISCUSSION} \ldots \ldots \ldots \ldots \ldots \ldots \ldots \ldots \ldots \ldots \ldots \ldots \ldots \ldots \ldots \ldots \ldots \ldots \ldots \ldots \ldots$

SCOPE AND PURPOSE $\ldots \ldots \ldots \ldots \ldots \ldots \ldots \ldots \ldots \ldots \ldots \ldots \ldots$

PRIOR WORK $\ldots \ldots \ldots \ldots \ldots \ldots \ldots \ldots \ldots \ldots \ldots \ldots \ldots \ldots \ldots \ldots$

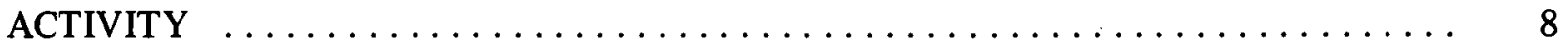

Surface Background Quality and Defect Characteristics $\ldots \ldots \ldots \ldots \ldots$

Optical Detection and Analysis of Isolated Defects, and Requirements

of an Optical System for Automated Inspection $\ldots \ldots \ldots \ldots \ldots \ldots \ldots \ldots$

System for Automated Inspection and Simple Analysis of Diffraction

Pattern Sizes Generated by Isolated Defects $\ldots \ldots \ldots \ldots \ldots \ldots \ldots \ldots \ldots \ldots \ldots$

Analysis of Isolated Defect Diffraction Pattern Sizes Based on the Diffraction Theory of Image Formation $\ldots \ldots \ldots \ldots \ldots \ldots \ldots \ldots \ldots$

Size of Diffraction Pattern Generated by an Isolated Pit Defect

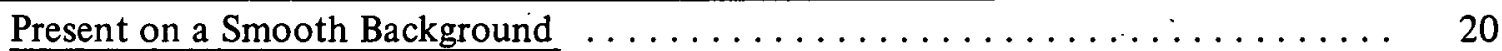

Size of Diffraction Pattern Generated by an Isolated Scratch Defect

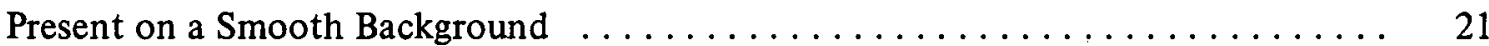

Proof That the Defect Diffraction Pattern in the Image Plane of a

Lens is Independent of Defect Position in the Illuminated Spot

Alternate Photodetector Arrangement for Exposing Central Maximum of Defect Pattern and Minimizing Diffuse Light From "Rough"

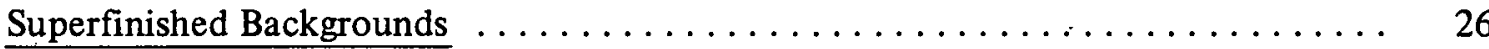

Diffracted Light Intensity as a Function of Isolated Defect Depth $\ldots \ldots \ldots \ldots$

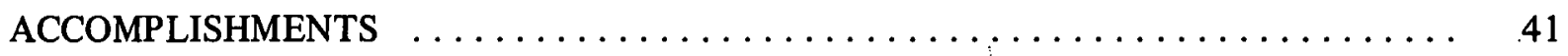

FUTURE WORK $\ldots \ldots \ldots \ldots \ldots \ldots \ldots \ldots \ldots \ldots \ldots \ldots \ldots \ldots \ldots \ldots \ldots$ 


\section{APPENDICES}

A. TRANSFORMATION OF LIGHT WAVE ELECTRIC FIELD AMPLITUDE FROM ONE TRANSVERSE PLANE TO ANOTHER . . . . . . 45

B. LIGHT WAVE ELECTRIC FIELD TRANSFORMATION FROM OBJECT PLANE TO IMAGE PLANE OF A LENS

C. LIGHT WAVE ELECTRIC FIELD IN THE IMAGE PLANE OF THE CENTER OF SPHERE IN TERMS OF THE ILLUMINATED SURFACE TRANSMISSION FUNCTION

D. DIFFRACTED INTENSITY VERSUS ISOLATED SCRATCH DEPTH

E. DIFFRACTED INTENSITY VERSUS ISOLATED PIT DEPTH FOR PIT WITH CONSTANT CONE ANGLE

F. DIFFRACTED INTENSITY VERSUS ISOLATED PIT DEPTH FOR PIT WITH VARIABLE CONE ANGLE 


\section{ILLUSTRATIONS}

Figure

Page

1 Schematic Representation of Superfinished Spherical Surface Profile Showing the Highly Reflective Background and Cross-Sections of Two Isolated Pit or

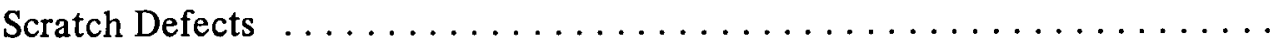

2 Arrangement Used to Detect and Measure Intensity .Distributions in Diffraction Patterns Produced by Illuminating Isolated Pit and Scratch Defects on Superfinished Spheres

3 Optical Arrangement for Displaying Reflected Light Defect Diffraction Pattern Centered About a Fixed Point P, Regardless of Defect Position in

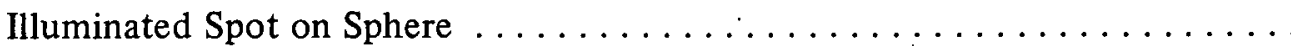

$4 \quad$ Illustration of Simple Analysis Used to Determine Defect

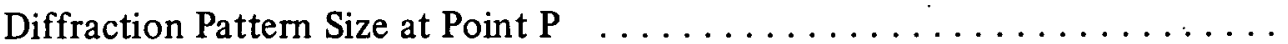

5 Geometry Illustrating Abbe Diffraction Theory of Image Formation $\ldots \ldots \ldots$

6 Geometry Used in Proof That Defect Diffraction Pattern Position in Plane at $S_{3}$ in Figure 3 is Independent of Defect Position in Illuminated Spot on Sphere

7 Schematic Comparisons of Isolated Pit and Scratch Diffraction Pattern Intensities With Focused Spot of Specular Light Intensity in the

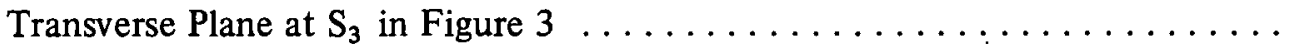

8 Schematic Representations of Light Signals in Plane at $S_{\mathbf{3}}$ for Isolated

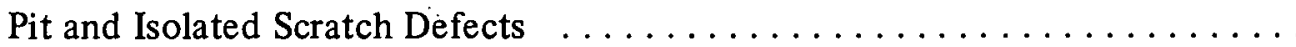

9 Possible Photodetector Array for Analyzing Defect Diffraction Patterns in a Transverse Plane Between $S_{3}$ Plane Lens of Focal Length $F$ in

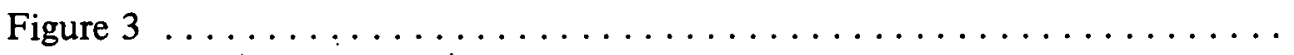

10 Geometry Used in Determining Diffraction Pattern Intensity Distribution From an Isolated Scratch Defect on a Superfinished Background ........

11 Geometry Used in Determining Diffracted Light Intensity Distribution From an Isolated Pit of Diameter 2R and Depth $h$ Present on a Superfinished. Background 
12 Comparison of Calculated Position of Pit Diffraction Pattern First Minimum Based on Diffracted Light From Model Pit in Figure 11, and Fraunhofer Diffraction by a Circular Aperture of Diameter Equal

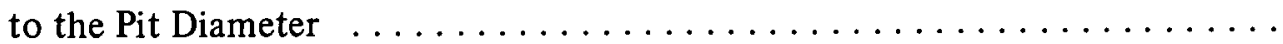

13 Cross-Section of a Model Pit Having Sides Which are Portions of Cones

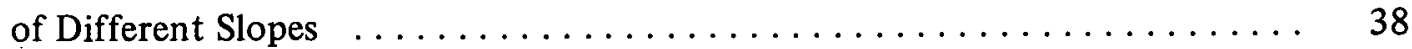

14 Calculated First Relative Maximum in Diffraction Pattern From Model Pit in Figure 13 Versus Maximum Pit Depth $h_{1} \ldots \ldots \ldots \ldots \ldots \ldots \ldots \ldots$

A-1 Light Wave Electric Field at a Source Point $P_{s}$ and at a Point $P$ at Distance $\mathrm{r}$ From $\mathrm{P}_{\mathrm{s}}$

A-2 Geometry Relating to the Light Wave Electric Field Transformation From Plane $\left(X_{1}, Y_{1}, Z_{1}\right)$ to Plane $\left(X_{2}, Y_{2}, Z_{1}+L\right)$

B-1 Object and Image Planes for a Thin Lens $\ldots \ldots \ldots \ldots \ldots \ldots \ldots \ldots \ldots$

B-2 Geometry Used in Obtaining the Form of Equation of Equation B-14 $\ldots \ldots \ldots 3$ 


\section{SUMMARY}

An optical arrangement was designed for use in a system being developed for automated inspection of superfinished spherical surfaces. Light from a helium neon laser is passed through a spatial filter to remove unwanted intensity variations in the beam profile, and the light proceeding from the filter is collimated by a lens of appropriate focal length. A second lens is used to converge the collimated light to a virtual focal point at the center of the sphere to be inspected. The geometry is such that all the rays incident on the highly reflective spherical surface are reflected back along their paths, and the retroreflected light is diverted by a beamsplitter. A third lens is used to focus the diverted light to a point on the face of a photodetector array capable of measuring light intensity as a function of radial and angular coordinates.

.As the sphere is rotated during the proposed automated scanning of the surface, a defect will pass into the illuminated spot on the sphere and a characteristic diffraction pattern will be observed in the plane of the detector array. It was shown theoretically and verified experimentally that the defect diffraction pattern is centered about the focused spot on the detector for any position of the defect within the illuminated spot on the sphere. This is a necessary requirement for rapid, practical automated scanning, detection, and analysis of surface defects because, during scanning, a defect will in general appear at an arbitrary position within the illuminated spot.

A theoretical relation was derived which relates the geometry of isolated pit and scratch defect diffraction patterns to the sizes of the defects and the dimensions of the optical system.

Experimental verification of the theory was obtained for a diffraction pattern generated by a $36-\mu \mathrm{m}$-diameter pit on a superfinished nickel sphere. Theoretical expressions were also derived which give the intensity of the defect-generated diffraction patterns in terms of the sizes of the isolated defects. The theory relating defect diffraction pattern sizes and intensities to the isolated defect sizes and the optical system geometry will aid in determining defect sizes from measured diffraction pattern intensities and thereby provide a basis for establishing surface certification . criteria for the automated inspection.system. 


\section{DISCUSSION}

\section{SCOPE AND PURPOSE}

An optical system for illuminating defects on superfinished spheres and appropriately displaying the resulting defect diffraction patterns to facilitate automated analysis of the pattern intensities was designed, analyzed theoretically, and tested. The work was conducted during the period of August 1974 through October 1975.

\section{PRIOR WORK}

Several techniques were investigated earlier as possible candidates for automated surface quality certification of superfinished metallic spheres. ${ }^{1}$ Surface waviness in a size range of concern on the spheres was detected and measured with a commercial laser interferometric displacement measurement system. Both specular and diffuse reflected light components were measured by illuminating the spheres with helium neon laser light and directing the reflected light onto photodetector arrangements. ${ }^{2}$

Measurements of the angular distribution of diffuse reflected light from a superfinished surface were used to calculate the average heights and slopes of the minute, randomly oriented background surface irregularities remaining after final polishing. A special light pipe array was constructed and used to measure the relatively large angular distribution of diffuse light from isolated pit and scratch defects in a size range of concern on superfinished spheres. ${ }^{3}$

The diffraction pattern intensities generated at small angles off the specular direction by illuminated isolated defects were measured with a commercial photodetector array. Both the angularly distributed diffuse light intensities and diffraction pattern intensities were correlated with the isolated defect dimensions.

\section{ACTIVITY}

Surface Background Quality and Defect Characteristics

The techniques of lapping and polishing are used to achieve highly reflective spherical metal surfaces which have very smooth profiles or "backgrounds." The surfaces often contain a few isolated defects in the form of pits and scratches as indicated in cross-section view in Figure 1. The superfinished surfaces obtained by the polishing techniques are superior to the normally available surfaces of precision ball bearings. The arithmetic average (AA) value of a typical superfinished surface background is a few tenths of one microinch (a few nanometers) or less. The small scale 

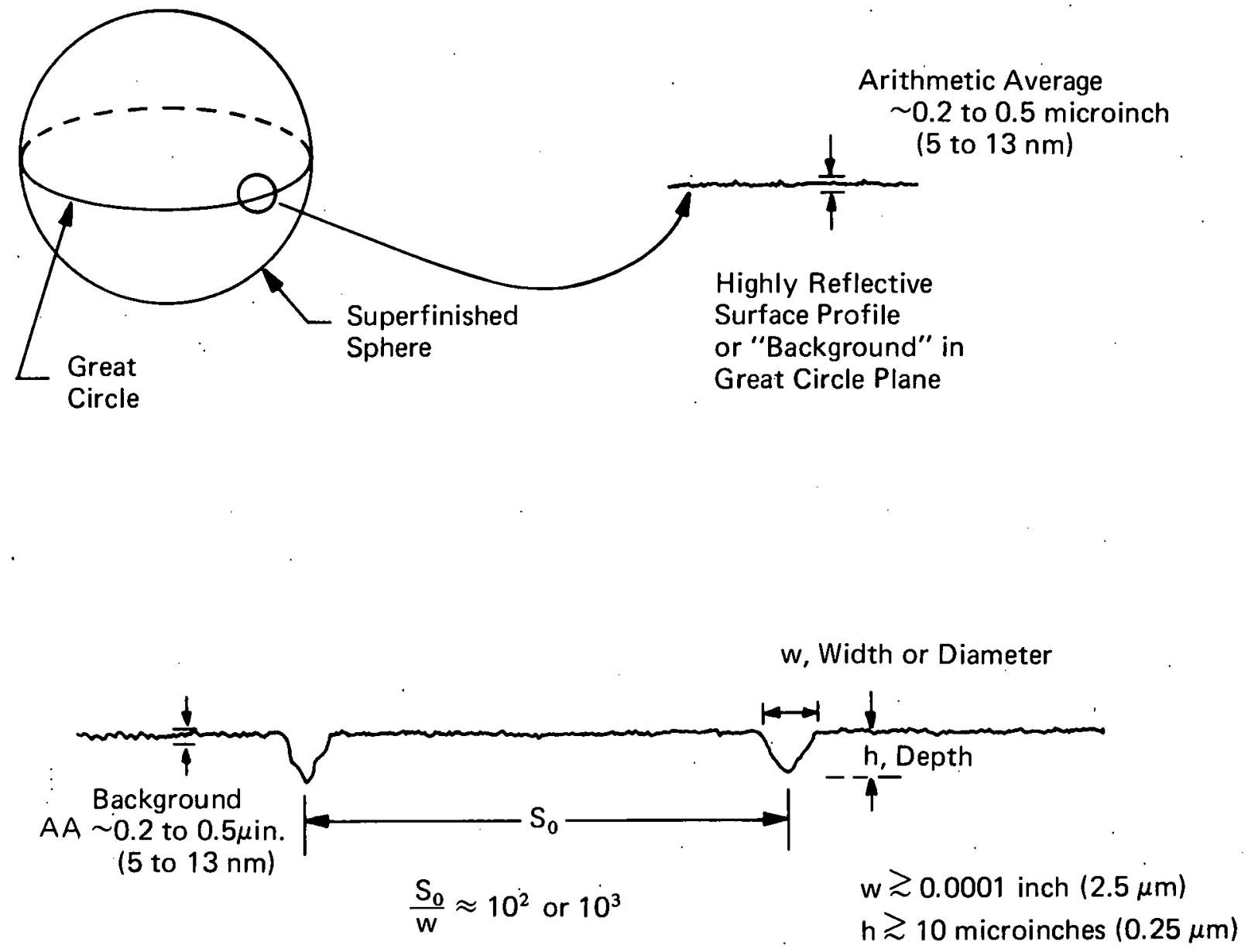

Figure 1. Schematic Representation of Superfinished Spherical Surface Profile Showing the Highly Reflective Background and Cross-Sections of Two Isolated Pit or Scratch Defects 
background roughness is a result of the minute, randomly oriented scratches and undulations remaining on the surface after final polishing. The pit and scratch defects are typically in the diameter or width range of 2.5 to $25 \mu \mathrm{m}$ and depth range of 0.25 to $5 \mu \mathrm{m}$. The defects are truly isolated in the sense that the interdefect spacing is typically two to three orders of magnitude greater than the defect diameter or width. It is necessary to inspect the surfaces to certify the background quality and to characterize the kinds and sizes of isolated defects present.

Optical Detection and Analysis of Isolated Defects, and Requirements of an Optical System for Automated Inspection

When a laser beam is incident on a highly reflective spherical surface as shown in Figure 2, the reflected beam can be intercepted by a screen and used to detect the presence of isolated defects on the sphere. If the beam is nearly normally incident on a defect-free region of the surface, the intensity of the spot on the screen is nearly symmetric about the center and decreases monotonically outward from the center. This is true for a Gaussian laser beam intensity profile incident on a highly reflective surface background containing only minute, randomly oriented irregularities. When an isolated pit on the surface is illuminated by the incident beam, a diffraction pattern consisting of alternately bright and dark concentric rings is observed on the screen. The pattern is qualitatively similar to Fraunhofer diffraction from a circular aperture. When an isolated scratch defect is illuminated, an alternately bright and dark parallel line diffraction pattern, qualitatively similar to classical diffraction from a narrow slit, is observed on the screen.

Earlier work showed that measurements of the diffraction pattern intensities in the plane of the screen could be correlated with dimensions of the isolated defects. ${ }^{2}$ In the earlier work a commercial multi-element photodetector array was positioned with its plane in the plane of the screen in Figure 2. The commercial photodetector array shown schematically in Figure 2 has a total active area in the form of a circle $31.8 \mathrm{~mm}$ in diameter which contains 32 semi-annular elemental ring detectors and an equal number of angular wedge detectors. The ring and wedge detectors are convenient for measuring radial and angular intensity distributions, respectively, relative to the center of the array.

Intensity measurements of a pit or scratch defect diffraction pattern were made only after positioning the sphere so that the defect was centered within the illuminated spot on the sphere. Under these conditions the ring diffraction pattern from a pit was centered about the detector center and therefore the symmetry of the detector array could be used advantageously. The successful results of the study indicated that the commercial photodetector array would be convenient for automated surface finish certification, provided the diffraction patterns could always be centered on the photodetector. However, with the system in Figure 2 the position of the diffraction pattern on the screen is dependent upon the position of the defect within the illuminated spot; therefore this system could not be used in automated surface inspection because . only by accident would an illuminated defect be centered in the illuminated spot. 


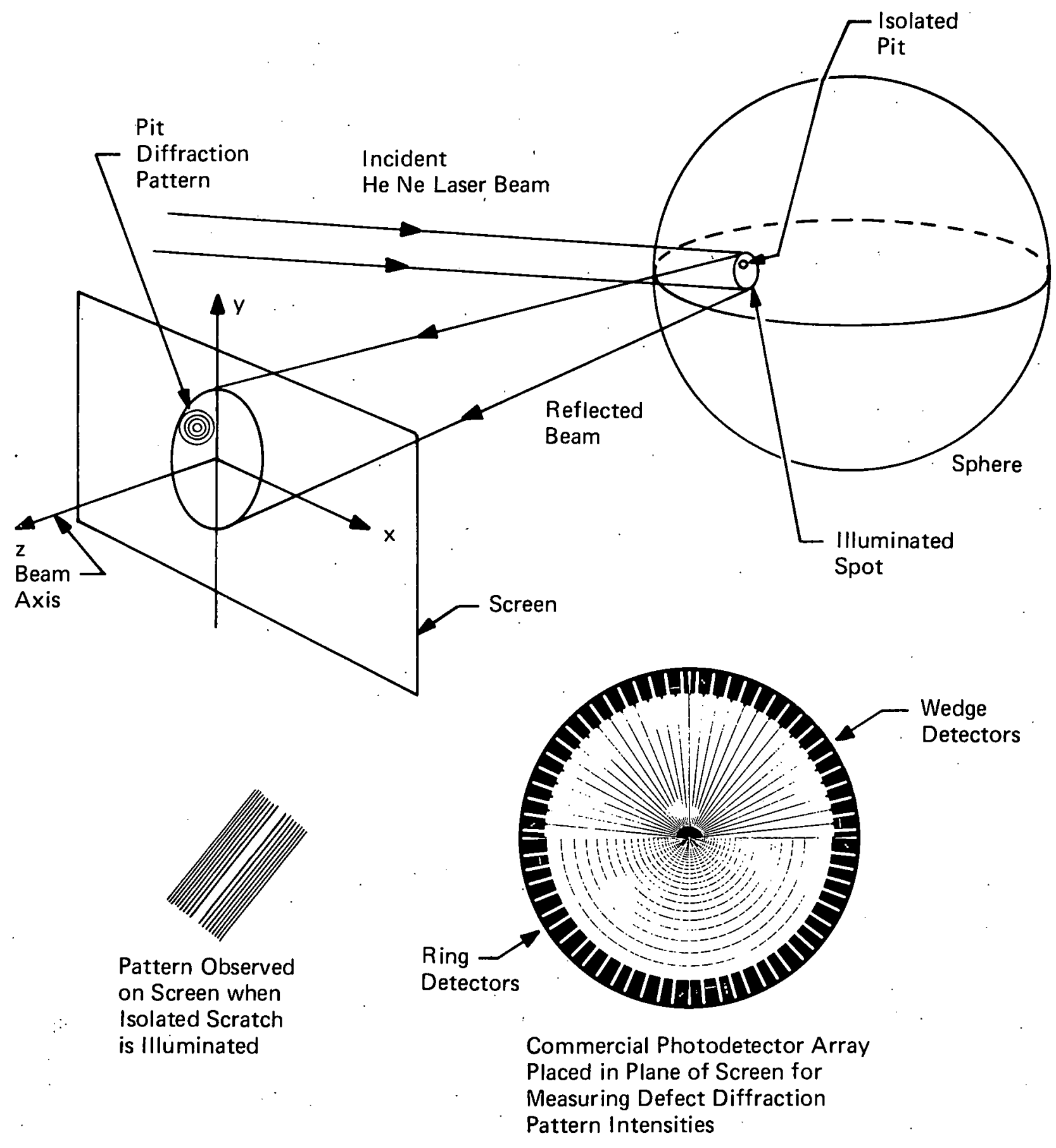

Figure 2. Arrangement Used to Detect and Measure Intensity Distributions in Diffraction Patterns Produced by Illuminating Isolated Pit and Scratch Defects on Superfinished Spheres (The defect diffraction pattern position on the screen depends on the position of the defect within the illuminated spot.) 
To facilitate automated analysis of the defect diffraction patterns, an optical system is required which will produce a defect diffraction pattern the location of which is independent of the defect position within the illuminated spot. An optical system for achieving this is described below and a theoretical analysis is presented relating the diffraction pattern intensities to the dimensions of the optical arrangement and the sizes of isolated defects.

System for Automated Inspection and Simple Analysis of Diffraction Pattern Sizes Generated by Isolated Defects

The proposed optical system for centering the defect diffraction patterns about a point $P$ in space is shown schematically in Figure 3. Light from a helium neon laser is passed through a spatial filter to remove unwanted intensity variations in the beam, and the light proceeding from the filter is collimated by a lens of appropriate focal length. A lens of focal length $f$ is used to focus the collimated light to a virtual focal point at the center of the test sphere. All the rays incident on the highly reflective spherical surface are reflected back along their paths, and the retroreflected light is diverted through 90 degrees by the beamsplitter. A lens of focal length $F$ is used to focus the diverted light to a point on the face of a photodetector array capable of measuring light intensity as a function of radial and angular coordinates.

Consider a single defect (pit or scratch) on the spherical surface and let it have a dimension $2 \mathbf{r}_{0}$ where $r_{0}$ is the pit radius or scratch half-width. It is assumed that $2 r_{0}$ is small in comparison with the illuminated spot on the sphere, which is equivalent to neglecting the finite collimated beam diameter. Since $2 r_{0}$ is very small in comparison with the beam size, the rays incident on the pit are virtually all parallel and therefore the pit can be regarded as an aperture which produces a virtual Fraunhofer diffraction pattern at the center $O$ of the sphere as shown in Figure 4. According to Fraunhofer diffraction theory, ${ }^{4}$ the intensity variation along line ab in the virtual Fraunhofer pattern is proportional to the square of the quantity

$\frac{\mathrm{r}_{0} \lambda}{\sin \theta} \mathrm{J}_{1}\left(\frac{2 \pi \mathrm{r}_{0} \sin \theta}{\lambda}\right)$

where $J_{1}$ is the first order Bessel function, $\theta$ is the angle between the lines joining the defect center with point $O$ and with point $O_{r}$ at which the intensity is to be evaluated, and $\lambda$ is the incident light wavelength. The lens of focal length $F$ forms at point $P$ a real image of the virtual Fraunhofer diffraction pattern at $\mathrm{O}$. From simple geometrical optics for thin lenses, the relation

$\frac{1}{S_{1}+S_{2}}+\frac{1}{S_{3}}=\frac{1}{F}$

holds in which $S_{1}$ is the distance from $O$ to the beamsplitter and $S_{2}$ is the distance from the beamsplitter to the lens of focal length $\mathrm{F}$. The size of the image at $\mathrm{P}$ bears a magnification $\mathrm{M}^{\prime}$ relative to the virtual image size at $O$, where the absolute value of $M^{\prime}$ is given by 


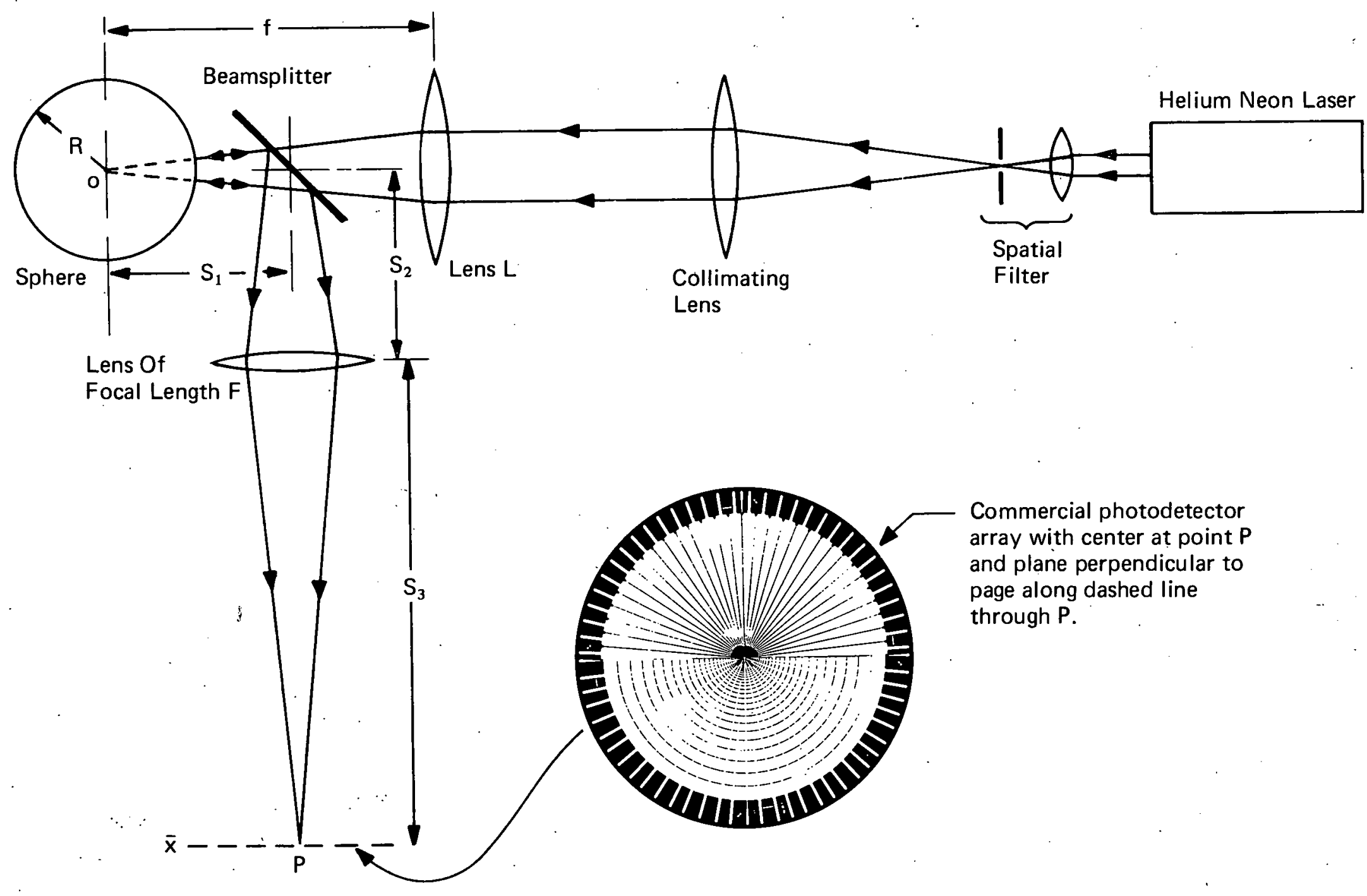

Figure 3. Optical Arrangement for Displaying Reflected Light Defect Diffraction Pattern Centered About a Fixed Point P, Regardless of Defect Position in Illuminated Spot on Sphere 


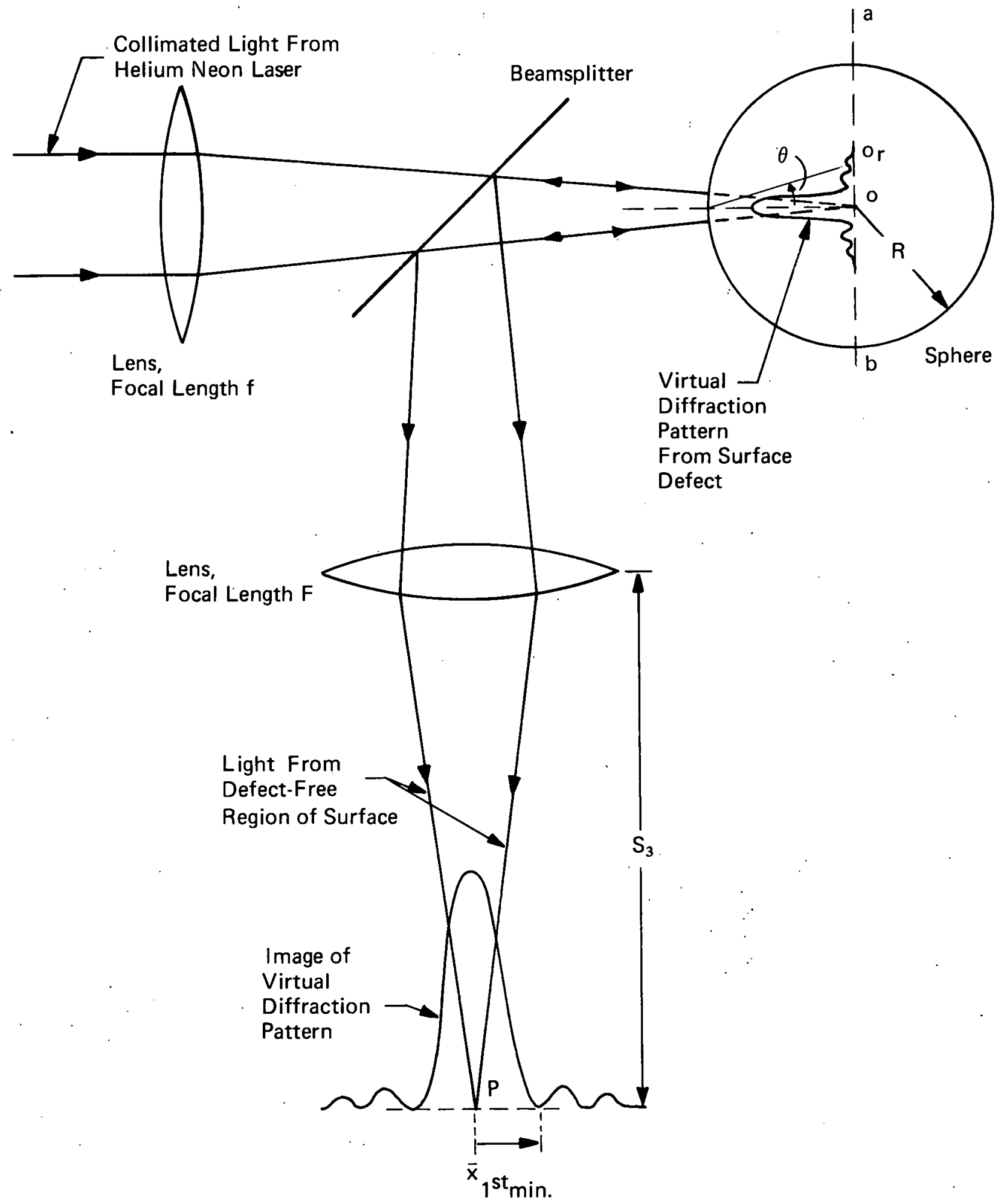

Figure 4. Illustration of Simple Analysis Used to Determine Defect Diffraction Pattern Size at Point $\mathbf{P}$ 
$\mathrm{M}^{\prime}=\frac{\mathrm{S}_{3}}{\mathrm{~S}_{1}+\mathrm{S}_{2}}$

Consider an arbitrary point $\mathrm{O}_{\mathrm{r}}$ on line $\mathrm{ab}$ at distance $\mathrm{r}$ from the center $\mathrm{O}$ of the virtual diffraction pattern at the center of the sphere in Figure 4. The corresponding point in the real-image diffraction pattern at $\mathrm{P}$ will occur at a distance $\overline{\mathrm{x}}$ from $\mathrm{P}$ given by

$\overline{\mathrm{x}}=\frac{\mathrm{S}_{3}}{\mathrm{~S}_{1}+\mathrm{S}_{2}} \mathrm{r}$.

For example, the first minimum in the virtual pattern occurs at the first zero of the Bessel function which, according to Bessel function tables, means that $\left(2 \pi \mathrm{r}_{0} \sin \theta\right) / \lambda=1.2197 \pi$. Therefore, the first minimum or dark band in the pattern at $P$ appears at a value $\bar{x}$ from Equation 4 given by

$\overline{\mathrm{x}}_{1} \mathrm{st}_{\min }=\frac{\mathrm{S}_{3}}{\mathrm{~S}_{1}+\mathrm{S}_{2}} \frac{1.2197 \lambda}{2 \mathrm{r}_{0}} \mathrm{R}=\frac{\left(\mathrm{S}_{3}-\mathrm{F}\right) 1.2197 \lambda}{\mathrm{F} 2 \mathrm{r}_{0}} \mathrm{R}$,

where the approximation $\sin \theta \approx \tan \theta \approx \theta=r / R$ was made because the angle $\theta$ is very small. Similarly, use of Bessel function tables shows that the distances to the second and third minima and the first and second relative maxima in the diffraction pattern centered at point $\mathrm{P}$ are given by the following expressions.

$$
\begin{aligned}
& \overline{\mathrm{x}}_{1} \mathrm{st}_{\max }=\frac{\mathrm{S}_{3}}{\mathrm{~S}_{1}+\mathrm{S}_{2}} \frac{0.819 \lambda}{\mathrm{r}_{0}} \mathrm{R} \\
& \bar{x}_{2} \text { nd }_{\min }=\frac{S_{3}}{S_{1}+S_{2}} \frac{1.116 \lambda}{r_{0}} R \\
& \bar{x}_{2^{\text {nd }}}=\frac{S_{3}}{S_{1}+S_{2}} \frac{1.333 \lambda}{r_{0}} R \\
& \bar{x}_{3} \mathrm{rd}_{\min }=\frac{\mathrm{S}_{3}}{\mathrm{~S}_{1}+\mathrm{S}_{2}} \frac{1.619 \lambda}{\mathrm{r}_{0}} \mathrm{R}
\end{aligned}
$$

Similarly, when an isolated scratch defect of length $L$ and width $w$ is illuminated, a virtual Fraunhofer diffraction pattern, characteristic of diffraction by a rectangular aperture with dimensions $\mathrm{LXw}$, is formed at the center of the sphere. If the scratch length $\mathrm{L}$ is much longer than the width $w$, then the virtual diffraction pattern at the center of the sphere consists essentially of a very narrow "line" diffraction pattern with a maximum at the center and relative maxima and minima located symmetrically on either side. According to Fraunhofer diffraction theory, the first minimum in the virtual pattern occurs at a distance ${ }^{4}$ 
$\mathrm{x}_{1} \mathrm{st}_{\min }=\mathrm{R} \frac{\lambda}{\mathrm{w}}$

from the center. The second minimum occurs at distance $2 R \lambda / w$ from the center and there is a relative maximum approximately midway between the two minima. Therefore, the distance to the first minimum in the scratch diffraction pattern at point $P$ in Figure 3 is

$\bar{x}_{1} s_{\min }=M^{\prime} R \frac{\lambda}{w}=\frac{S_{3}-F}{F} R \frac{\lambda}{w}$.

Analysis of Isolated Defect Diffraction Pattern Sizes Based on the Diffraction Theory of Image Formation

The general result for a pit in Equation 5 can also be obtained by an analysis of the light wave amplitudes in various planes perpendicular to the optical axis of the system. This analysis is based on the Abbe diffraction theory of image formation with coherent light. ${ }^{4}$ According to this theory the virtual focal point at the center of the sphere in Figure 5 acts as a source of light. If there are no diffracting obstacles within the illuminated spot on the sphere, the light will be focused to a "perfect" spot (limited only by diffraction from finite beam or aperture sizes) at a distance $\mathrm{S}_{\mathbf{3}}$ from the lens. That is, the virtual point object at the center of the sphere is imaged in the transverse plane at $S_{3}$. However, the presence of a diffracting obstacle, such as a defect, alters the nature of the transmission function representing the illuminated spot on the sphere. As a result, some of the virtual light is diffracted away from its simple geometric path as it passes "through" the surface. The light diffracted at a point $\mathrm{x}_{0}, \mathrm{y}_{0}$ is focused by the lens to point $\overline{\mathrm{x}}, \overline{\mathrm{y}}$ at a distance $\sqrt{\overline{\mathrm{x}}^{2}+\overline{\mathrm{y}}^{2}}$ from the optical axis in the transverse plane at distance $S_{3}$ from the lens. The diffracted rays, which are focused in the plane at $S_{3}$ pass on beyond this plane and form an image of the illuminated spot on the sphere, in the transverse plane at distance $S$ from the lens.

The virtual focal point $O$ at the center of the sphere can be considered a point source which emits spherical light waves of amplitude $E_{0}$ and wavelength $\lambda$ whose propagation is described by the function

$\mathrm{E}=\frac{1}{\ell} \mathrm{E}_{0} \mathrm{e}^{-\mathrm{i} \frac{2 \pi \ell}{\lambda}}$

where $\ell$ is the radial distance from the source point $O$. The distance between $O$ and a point with coordinates $\mathrm{x}_{0}, \mathrm{y}_{0}$ in the plane perpendicular to the axis at the position of the small illuminated spot is $\ell=\left(R^{2}+x_{0}^{2}+y_{0}^{2}\right)^{1 / 2}$ which can be approximated by $\ell \approx R+\left(x_{0}^{2}+y_{0}^{2}\right) / 2 R \approx R$ for $\mathrm{x}_{0}, \mathrm{y}_{0}<<\mathrm{R}$. The illuminated circular region on the surface of the sphere can be assumed to lie wholly within the $\mathrm{x}_{0}, \mathrm{y}_{0}$ plane tangent to the sphere at the center of the illuminated spot because the spot diameter is very much smaller than the sphere diameter. Therefore, the light wave leaving 


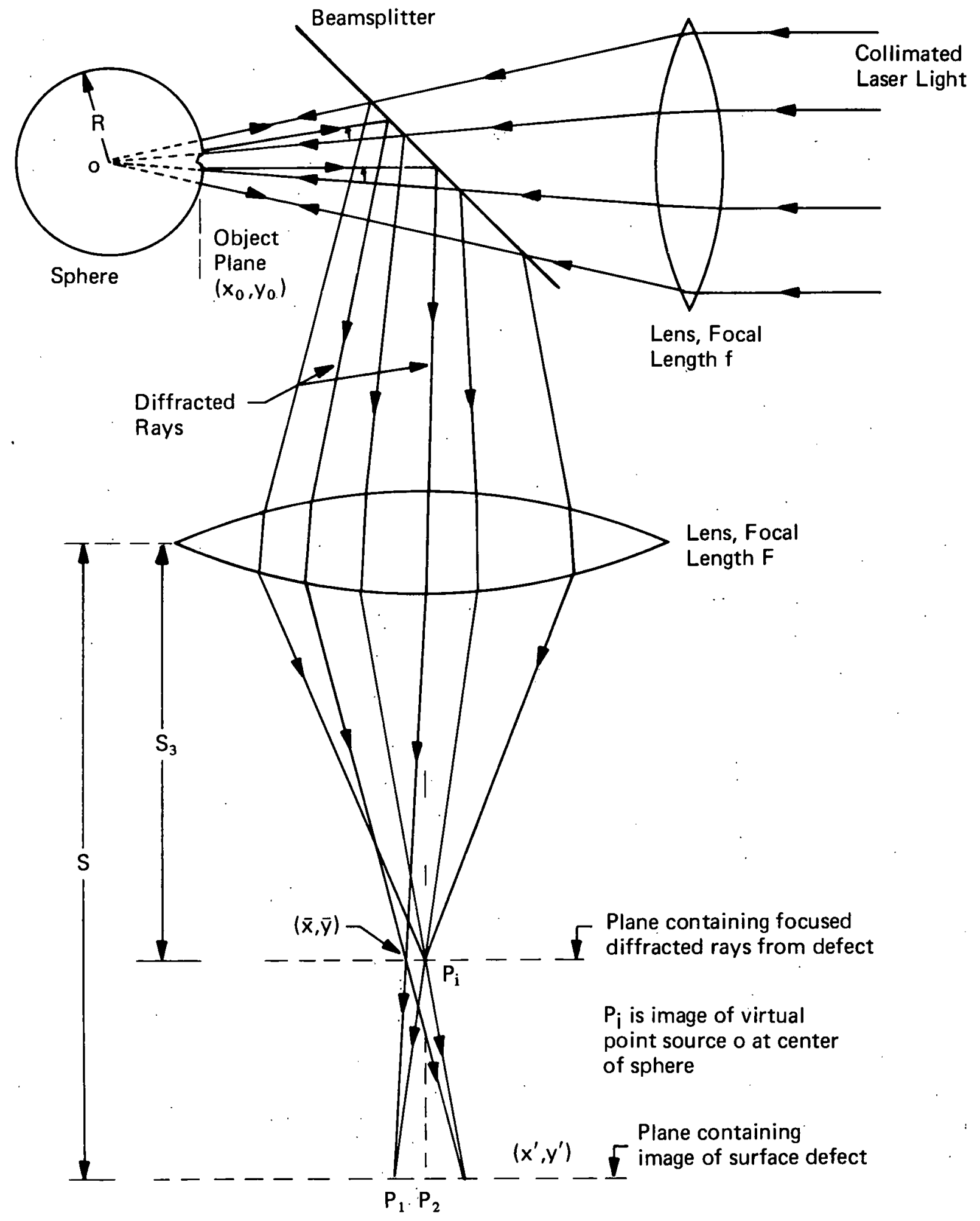

Figure 5. Geometry Illustrating Abbe Diffraction Theory of Image Formation 
the point $O$ and described by Equation 10 will be represented by the function

$E=\frac{E_{0}}{R} e^{-i \frac{2 \pi}{\lambda} R} e^{-i \frac{2 \pi}{\lambda} \frac{x_{0}^{2}+y_{0}^{2}}{2 R}}$

as it arrives at the position of the illuminated spot. After passing through the spherical surface-air boundary, the light wave will be modulated by the surface, and, just after passage through the surface, the light wave amplitude is

$E\left(x_{0}, y_{0}, R\right)=\left[\frac{E_{0}}{R} e^{-i \frac{2 \pi}{\lambda} R} e^{-i \frac{2 \pi}{\lambda} \frac{x_{0}^{2}+y_{0}^{2}}{2 R}}\right] \quad T\left(x_{0}, y_{0}\right)$

where $T\left(x_{0}, y_{0}\right)$ is the transmission function of the illuminated spherical surface.

According to the electric field transformation derived in Appendix B which gives the field in the image plane of a thin lens in terms of the electric field in the source or object plane (opposite side of lens) of the lens, the light wave field in the image plane of Figure 3, under the assumption of an infinite lens aperture, can be obtained from the electric field expression in Equation 12.

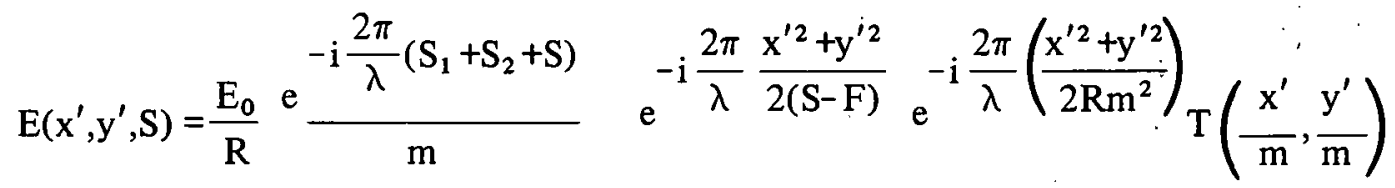

The coordinates $x^{\prime}, y^{\prime}$ measure the position of a point in the transverse plane at distance $S$ from the lens of focal length $F$ in Figure 5 . The quantity $m$ is the magnification and its absolute value is $|\mathrm{m}|=\mathrm{x}^{\prime} / \mathrm{x}_{0}=\mathrm{y}^{\prime} / \mathrm{y}_{0}$. The phase shift

$\exp \left\{-\mathrm{i} \frac{\pi}{\lambda}\left[\mathrm{x}^{\prime 2}+\mathrm{y}^{\prime 2}\right]\left(\frac{\mathrm{l}}{\mathrm{Rm}^{2}}+\frac{1}{(\mathrm{~S}-\mathrm{F})}\right)\right\}$

in the overall exponential factor of Equation 13 is the shift caused by the propagation from $O$ to $P_{1}$ minus that caused by propagation from $\mathrm{O}$ to $\mathrm{P}_{2}$ in Figure 5. (There is no phase difference among the various optical paths from $O$ to $P_{i}$.) Since the coordinates of a point in the image plane are $\left(x^{\prime}, y^{\prime}\right)$, the use of the geometry in Figure 5 shows that equality between the optical path differences and the phase shift factor in Equation 13 leads to the relation derived in Appendix $C$,

$\frac{1:}{m^{2} R}+\frac{1}{S-F}=\frac{1}{S-S_{3}}$

and this expression allows Equation 13 to be written as 
$E\left(x^{\prime}, y^{\prime}, S\right)=\frac{E_{0}}{m R}\left[e^{-i \frac{2 \pi}{\lambda}\left(S_{1}+S_{2}+S\right)} e^{-i \frac{2 \pi}{\lambda} \frac{x^{\prime 2}+y^{\prime 2}}{2\left(S-S_{3}\right)}}\right] T\left(\frac{x^{\prime}}{m}, \frac{y^{\prime}}{m}\right)$.

This is the light wave electric field in the image plane in terms of the scaled transmission function $\mathrm{T}\left(\mathrm{x}^{\prime} / \mathrm{m}, \mathrm{y}^{\prime} / \mathrm{m}\right)$ of the illuminated spot on the spherical surface.

The light wave electric field in the image plane through point $\mathrm{P}$ can be obtained by performing a transformation of the type derived in Appendix A on the light wave electric field in the image plane given by Equation 14 . That is, the field at $\left(\bar{x}, \bar{y}, S_{3}\right)$ is given by the integral

$E\left(\bar{x}, \bar{y}, S_{3}\right)=\frac{i e^{-i \frac{2 \pi}{\lambda}\left(S_{3}-S\right)}}{\lambda\left(S_{3}-S\right)} \iint_{-\infty}^{\infty} e^{-i \frac{2 \pi}{\lambda} \frac{1}{2\left(S_{3}-S\right)} \cdot\left[\left(\bar{x}-x^{\prime}\right)^{2}+\left(\bar{y}-y^{\prime}\right)^{2}\right]} E\left(x^{\prime}, y^{\prime}, S\right) d x^{\prime} d y^{\prime}$

Substitution of Equation 14 into the integrand of Equation 15 gives

$E\left(\bar{x}, \bar{y}, S_{3}\right)=-\frac{i E_{0}}{m \lambda\left(S-S_{3}\right) R} e^{-i \frac{2 \pi}{\lambda}\left[S_{1}+S_{2}+S_{3}+\frac{\bar{x}^{2}+\bar{y}^{2}}{2\left(S_{3}-S\right)}\right] x}$
$\iint_{-\infty}^{\infty} T\left(\frac{x^{\prime}}{m}, \frac{y^{\prime}}{m}\right) e^{-\frac{2 \pi i}{\lambda\left(S-S_{3}\right)}\left(x^{\prime} \bar{x}+y^{\prime} \vec{y}\right)} d x^{\prime} d y^{\prime}$.

The intensity $I$ in the focal plane at point $P$ in Figure 3 is given by

$I=E\left(\bar{x}, \bar{y}, S_{3}\right) E^{*}\left(\bar{x}, \bar{y}, S_{3}\right)$

where $E^{*}$ denotes the complex conjugate of $E\left(\bar{x}, \bar{y}, S_{3}\right)$. Multiplication of $E\left(\bar{x}, \bar{y}, S_{3}\right)$ by its complex conjugate causes all the exponentials outside the integral in Equation 16 to cancel, and when the variables are expressed in terms of the coordinates in the illuminated spot, the following is obtained.

$I=\frac{m^{2} E_{0}^{2}}{R^{2} \lambda^{2}\left(S-S_{3}\right)^{2}}\left[\iint_{-\infty}^{\infty} T\left(x_{0}, y_{0}\right) e^{i \frac{2 \pi m}{\lambda\left(S-S_{3}\right)}\left(x_{0} \bar{x}+y_{0} \bar{y}\right)} d x_{0} d y_{0}\right]^{2}$

From the mathematical form of the double integral in Equation 17 it is seen that the intensity in the transverse plane at distance $S_{3}$ from the lens is proportional to the square of the Fourier transform of the transmission function representing the illuminated spot on the surface of the sphere in Figure 3. 
$\underline{\text { Size of Diffraction Pattern Generated by an Isolated Pit Defect Present on a Smooth Background }}$

In applying Equation 17 to calculate the intensity distribution at point $\mathrm{P}$ in Figure 3, the transmission function $T\left(x_{0}, y_{0}\right)$ representing the illuminated region of the spherical surface must be defined. If the illuminated region is defect free, the function $T\left(x_{0}, y_{0}\right)$ can be taken as a constant, and for convenience the constant can be set equal to unity. In this case the only diffraction effects observed at point $\mathrm{P}$ are those from the finite size of the beam. However, it is assumed that the beam size is sufficiently large that these diffraction effects occupy only a small part of the space and do not contribute measurable signals in the transverse plane at $S_{3}$. If only those diffraction effects caused by the presence of a defect within the illuminated region are to be considered, then $\mathrm{T}\left(\mathrm{x}_{0}, \mathrm{y}_{0}\right)$ can be taken as zero for the case of a defect-free illuminated spot.

Consider the case in which a single pit defect of diameter $2 r_{0}$ is present within the illuminated region of the highly reflective spherical surface. It is assumed that the illuminated spot diameter is much larger than the pit diameter, so that finite-size beam or aperture diffraction effects do not mask or interfere with the pit diffraction effects. In this case the transmission function can be taken as zero every where outside the pit boundary, and unity within the area $\pi r_{0}^{2}$ defined by the pit. For these conditions the evaluation of the double integral in Equation 17 is identical to that involved in calculating the Fraunhofer diffraction pattern intensities for light passing through a circular aperture. ${ }^{4}$ Therefore, the light intensity I from Equation 17, as a function of distance $\bar{x}$ outward from the optical axis in the transverse plane at $S_{3}$ in Figure 3, is proportional to the square of the first order Bessel function $\mathbf{J}_{\mathbf{1}}$.

$I \propto\left\{\mathrm{J}_{1}\left[\frac{2 \pi \mathrm{r}_{0} \mathrm{~m} \overline{\mathrm{x}}}{\lambda\left(\mathrm{S}-\mathrm{S}_{3}\right)}\right]\right\}^{2}$

The argument of the Bessel function contains only the coordinate $\bar{x}$ in the transverse plane at $S_{3}$ because the diffraction pattern in this plane is symmetric about the optical axis.

It can be shown that the dimensions of the pit diffraction pattern in the plane at $S_{3}$, as determined by the relation in Equation 17a, are identical to those derived earlier through the simple analysis leading to Equation 5. To show this, note that the first minimum in the pit diffraction pattern intensity I will occur when the argument of the Bessel function in Equation 17a has its first minimum. Thus, from Bessel function tables, the first minimum in the diffraction pattern will occur at a distance $\bar{x}$ from the center so that

$\frac{2 \pi \mathrm{r}_{0} \mathrm{~m} \overline{\mathrm{x}}_{1} \mathrm{st}_{\mathrm{min}}}{\lambda\left(\mathrm{S}-\mathrm{S}_{3}\right)}=1.2197 \pi$

or 
$\overline{\mathrm{x}}_{1} \mathrm{st}_{\min }=\frac{\mathrm{S}-\mathrm{S}_{3}}{\mathrm{~m}} \frac{1.2197}{2 \mathrm{r}_{0}} \lambda$

The distances $S_{1}, S_{2}, S_{3}$, and $S$ are related to the lens focal length $F$, the magnification $m$, and the sphere radius $R$ by the relations $m=S /\left(S_{1}+S_{2}-R\right), 1 / S+1 /\left(S_{1}+S_{2}-R\right)=1 / F$, and $1 / S_{3}+1 /\left(S_{1}+S_{2}\right)=1 / F$. When these relations are combined, it can be shown by considerable algebraic manipulation that Equation 18 can be expressed as

$\overline{\mathrm{x}}_{1} \mathrm{st}_{\min }=\frac{\mathrm{S}_{3}-\mathrm{F}}{\mathrm{F}} \frac{1.2197 \lambda}{2 \mathrm{r}_{0}} \mathrm{R}$,

which is identical with the result obtained earlier by the simpler analysis leading to Equation 5 . It follows that the other maxima and minima positions in the pattern at $\mathrm{P}$ will agree with the locations given earlier by Equations 6 through 9. Therefore, the two analyses of the defect diffraction pattern sizes at point $P$ give identical results.

In one arrangement of the optical system, an isolated pit of diameter $35 \mu \mathrm{m}$ present on a superfinished nickel sphere of radius $R=7.8 \mathrm{~mm}$ was illuminated. A lens with focal length $F=200 \mathrm{~mm}$ was used and the distance $S_{3}$ was $9.29 \mathrm{~m}$. The observed distance from the center to the first minimum in the diffraction pattern at point $P$ was $7.6 \mathrm{~mm}$. When the helium neon laser wavelength $\lambda=0.6328 \mu \mathrm{m}$ and the above values for $\mathrm{R}, \mathrm{S}_{3}, \mathrm{~F}$, and $\mathrm{r}_{0}$ are substituted into Equation 19 , the calculated value of the distance to the first minimum in the diffraction pattern at $P$ is $\bar{x}_{1}$ st ${ }_{\min }$ $=7.7 \mathrm{~mm}$, which is in good agreement with the experimental observation.

Size of Diffraction Pattern Generated by an Isolated Scratch Defect Present on a Smooth Background

Consider an isolated scratch of width $2 \mathrm{a}$ and length $2 \mathrm{~b}$ present on a highly reflective background. For this case the transmission function $T\left(x_{0}, y_{0}\right)$ in Equation 17 can be taken as unity within the rectangle $2 \mathrm{a} \times 2 \mathrm{~b}$ defining the scratch and as zero at all other points within the illuminated spot. The intensity $I_{s}$ in the transverse plane at $S_{3}$ is therefore proportional to the expression

$I_{s} \propto\left[\int_{x_{0}=-a}^{a} \int_{y_{0}=-b}^{b} e^{-i \frac{2 \pi m}{\lambda\left(S-S_{3}\right)}\left(x_{0} \bar{x}+y_{0} \bar{y}\right)} d x_{0} d y_{0}\right]^{2}$

which is identical to the integral for evaluating the Fraunhofer diffraction pattern intensity when light passes through a rectangular slit of dimensions $2 \mathrm{a} \times 2 \mathrm{~b}$. Therefore, the diffraction pattern intensity, calculated from Equation 20, is 


$$
I_{s}=\frac{16 m^{2} E_{0}^{2}}{R^{2} \lambda^{2}\left(S-S_{3}\right)^{2}} \cdot \frac{\sin ^{2}\left[\frac{2 \pi m \overline{x a}}{\lambda\left(S-S_{3}\right)}\right]}{\left[\frac{2 \pi m \bar{x}}{\lambda\left(S-S_{3}\right)}\right]^{2}} \cdot \frac{\sin ^{2}\left[\frac{2 \pi m \bar{y} b}{\lambda\left(S-S_{3}\right)}\right]}{\left[\frac{2 \pi m \bar{y}}{\lambda\left(S-S_{3}\right)}\right]^{2}} .
$$

It can be seen that at a constant value of $\bar{y}$ the intensity distribution is symmetrical about $\bar{x}=0$; likewise, at a constant value of $\bar{x}$, the intensity variations are symmetrical about the line $\bar{y}=0$. However, this is the extent of the scratch diffraction pattern symmetry, and it can be seen that an isolated scratch defect creates a diffraction pattern of lower symmetry than that produced by an isolated circular pit defect.

If the scratch is long compared with its width $(b>>a)$, the intensity variations associated with the $\bar{y}$ coordinate are very rapid relative to those in the $\overline{\mathrm{x}}$ direction. For this case the factor of the form $\frac{\sin \epsilon}{\epsilon}$ approaches a delta function $\delta(\bar{y})$, which is zero except at $\bar{y}=0$ where it is unity. The diffraction pattern intensity in the plane at $S_{3}$ is described adequately by the simplified form of Equation 21

$$
I_{s}=\frac{16 m^{2} E_{0}^{2}}{R^{2} \lambda^{2}\left(S-S_{3}\right)^{2}} \cdot \frac{\sin ^{2}\left[\frac{2 \pi m \bar{x} a}{\lambda\left(S-S_{3}\right)}\right]}{\left[\frac{2 \pi m \bar{x}}{\lambda\left(S-S_{3}\right)}\right]^{2}} \quad(b>>a)
$$

The first minimum in the pattern occurs at a distance

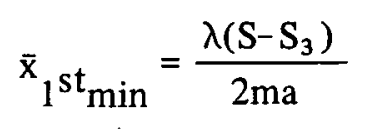

from the center. In general, minima occur on either side of $\bar{x}=0$ at distances given by

$\overline{\mathrm{x}}_{\min }=\frac{\mathrm{N} \lambda\left(\mathrm{S}-\mathrm{S}_{3}\right)}{2 \mathrm{ma}}$

where $\mathrm{N}=1,2,3, \cdots$. A relative maximum intensity occurs approximately midway between adjacent minima. It was noted earlier in obtaining Equation 19 that $\left(\mathrm{S}-\mathrm{S}_{3}\right) / \mathrm{m}=\mathrm{R}\left(\mathrm{S}_{3}-\mathrm{F}\right) / \mathrm{F}$, and therefore the minima positions in the transverse plane at $S_{3}$ in Figure 5 are obtained from the relation

$$
\overline{\mathrm{x}}_{\min }=\frac{\mathrm{N} \lambda\left(\mathrm{S}_{3}-\mathrm{F}\right)}{2 \mathrm{Fa}} \mathrm{R}
$$


Proof That the Defect Diffraction Pattern in the Image Plane of the Lens is Independent of Defect Position in the Illuminated Spot

Consider an isolated scratch of width $2 \mathrm{a}$ and length $2 \mathrm{~b}$ which is located at an arbitrary position within the illuminated spot on the spherical surface. The scratch will in general be inclined at an angle with the plane containing the sphere center and optical axis of the lens system. No loss in generality of the following argument will occur if, for convenience, the $\mathrm{x}_{0}, \mathrm{y}_{0}$ coordinate system in the plane of the small illuminated spot in Figure 5 is positioned so that one of the axes is parallel with the length dimension of the scratch. This situation is shown in Figure 6a. The limits on the double integral in Equation 16, which gives the light wave electric field $E\left(\bar{x}, \bar{y}, S_{3}\right)$ in the transverse plane at $S_{3}$, are therefore

$\cdot E\left(\bar{x}, \bar{y}, S_{3}\right)=\frac{-i E_{0} m e^{i \times \text { factor }}}{\lambda\left(S-S_{3}\right) R}\left[\int_{x_{0}=h_{1}-a}^{h_{1}+a} \int_{y_{0}=h_{2}-b}^{h_{2}+b} e^{-i \frac{2 \pi m}{\lambda\left(S-S_{3}\right)}\left[x_{0} \bar{x}+y_{0} \bar{y}\right]} d x_{0} d y_{0}\right]$

where the transmission function $\mathrm{T}\left(\mathrm{x}_{0}, \mathrm{y}_{0}\right)$ is taken as unity over the rectangular area of the scratch and as zero elsewhere. When the integration is performed in Equation 25 the result is as follows.

$$
\begin{aligned}
& \left.E\left(\bar{x}, \bar{y}, S_{3}\right)=\left.\frac{i E_{0} \lambda\left(S-S_{3}\right)}{4 m R \pi^{2} \bar{x} \bar{y}} e^{i \times \text { factor }\left\{\left.e^{-\frac{2 \pi i m}{\lambda\left(S-S_{3}\right)}} x_{0} \bar{x}\right|_{h_{1}-a} ^{h_{1}+a} e^{-\frac{2 \pi i m}{\lambda\left(S-S_{3}\right)}} y_{0} \bar{y}\right.}\right|_{h_{2}-b} ^{h_{2}+b}\right\} \\
& =\frac{i E_{0} \lambda\left(S-S_{3}\right)}{4 m R \pi^{2} \bar{x} \bar{y}} e^{i \times \text { factor }}\left\{e^{-\frac{2 \pi i m}{\lambda\left(S-S_{3}\right)} \bar{x} h_{1}}\left(e^{-\frac{2 \pi i m}{\lambda\left(S-S_{3}\right)} \bar{x} a}-e^{\frac{2 \pi i m}{\lambda\left(S-S_{3}\right)} \bar{x} a}\right)\right.
\end{aligned}
$$

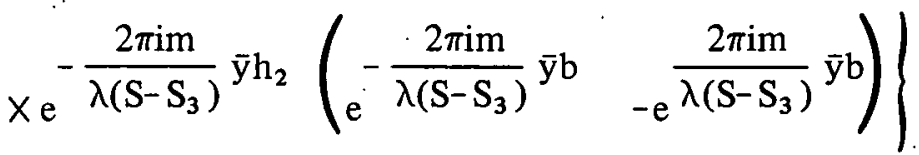

The intensity $I$ in the transverse plane at $S_{3}$ is obtained by forming the product of $E\left(\bar{x}, \bar{y}, S_{3}\right)$ with its complex conjugate $E^{*}\left(\bar{x}, \bar{y}, S_{3}\right)$. When this is done with the expression for $E\left(\bar{x}, \bar{y}, S_{3}\right)$ in Equation 26 the exponentials involving the arbitrary scratch position $\left(\mathrm{h}_{1}, \mathrm{~h}_{2}\right)$ cancel one another and the resulting relation for the intensity I becomes

$I_{S}=\frac{16 m^{2} E_{0}^{2}}{R^{2} \lambda^{2}\left(S-S_{3}\right)^{2}} \cdot \frac{\sin ^{2}\left[\frac{2 \pi m \bar{x} a}{\lambda\left(S-S_{3}\right)}\right]}{\left[\frac{2 \pi m \bar{x}}{\lambda\left(S-S_{3}\right)}\right]^{2}} \cdot \frac{\sin ^{2}\left[\frac{2 \pi m \bar{y} b}{\lambda\left(S-S_{3}\right)}\right]}{\left[\frac{2 \pi m \bar{y}}{\lambda\left(S-S_{3}\right)}\right]^{2}}$ 


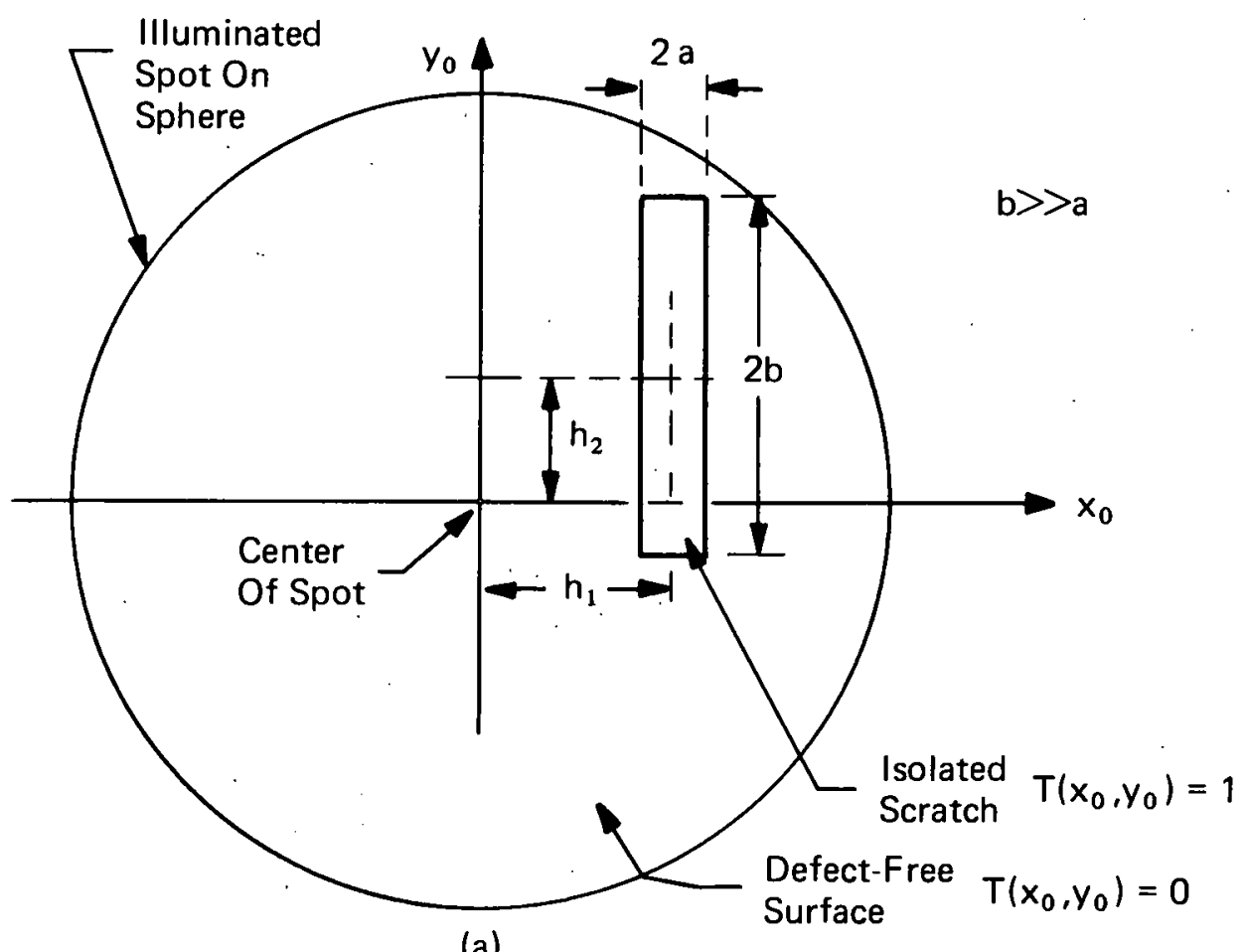

(a)

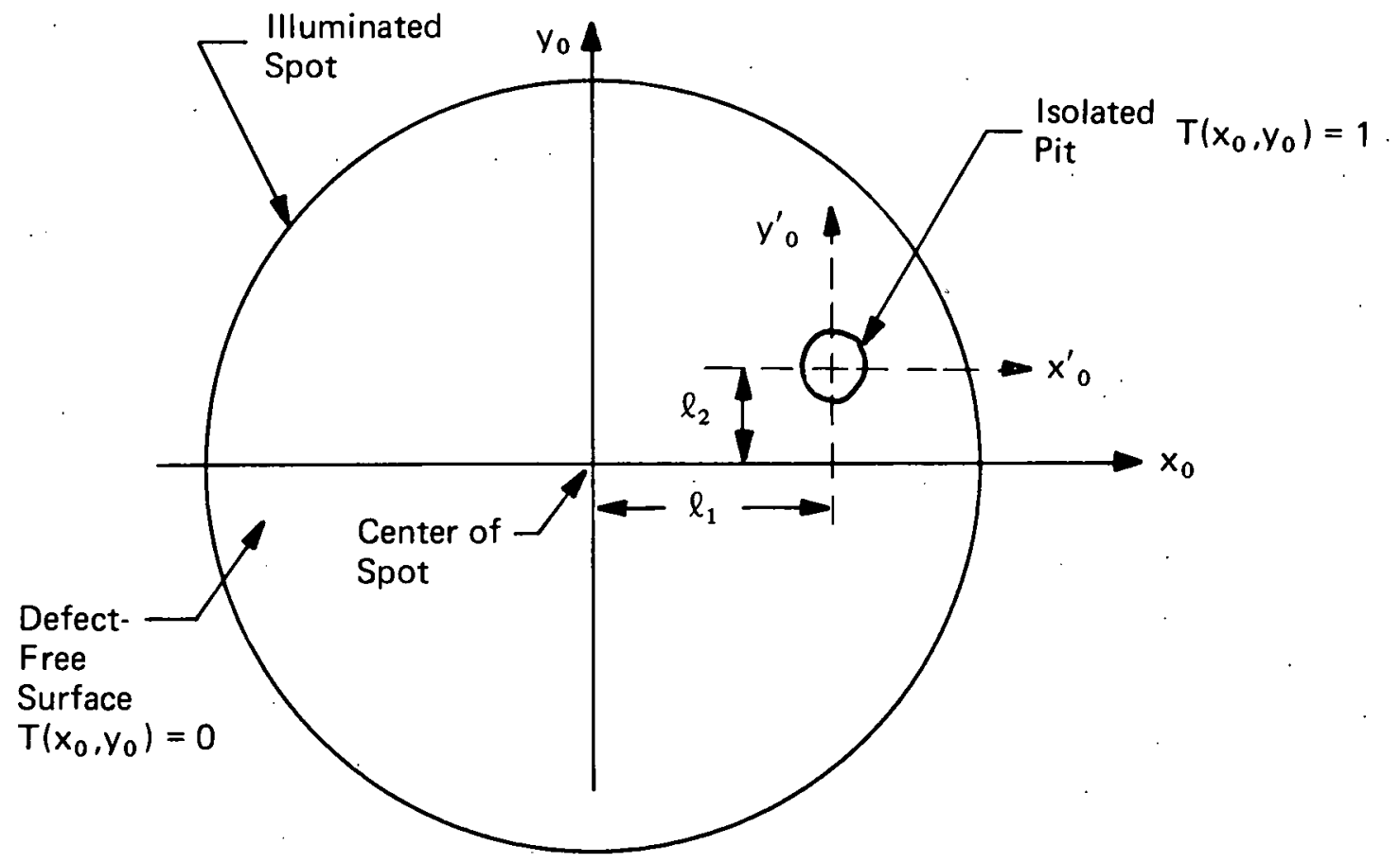

(b)

Figure 6. Geometry Used in Proof That Defect Diffraction Pattern Position in Plane at $S_{3}$ in Figure 3 is Independent of Defect Position in Illuminated Spot on Sphere 
where Euler's relation was used to convert the exponentials to sine and cosine form. The latter equation is identical to Equation 21 and indicates that the scratch defect diffraction pattern intensity in the transverse plane at $S_{3}$ is invariant with respect to the scratch position within the illuminated spot on the sphere.

Consider next the case in which an isolated pit defect, present on a smooth background surface, is arbitrarily located within the illuminated spot on the sphere as shown in Figure 6b. Let the center of the pit be located at the point with coordinates $\mathrm{x}_{0}=\ell_{1}, \mathrm{y}_{0}=\ell_{2}$ relative to the center of the $\mathrm{x}_{0} \mathrm{y}_{0}$ coordinate system. The center of the $\mathrm{x}_{0} \mathrm{y}_{0}$ coordinate system coincides with the center of the illuminated spot which lies on the optical axis of the system. Define a coordinate system $x_{0}^{\prime} y^{\prime}{ }_{0}$ with center at the pit center and oriented with its axes parallel, respectively, to the $x_{0}$ and $y_{0}$ axes as indicated in Figure 6b. When the coordinate transformations $\mathrm{x}_{0}^{\prime}=\mathrm{x}_{0}-\ell_{1}$ and $\mathrm{y}_{0}^{\prime}=\mathrm{y}_{0}-\ell_{2}$ relating the coordinates of a point in the $\mathrm{x}_{0}^{\prime} \mathrm{y}^{\prime}{ }_{0}$ system to its coordinates in the $\mathrm{x}_{0} \mathrm{y}_{0}$ system are used, Equation 16 can be put in the form

$E\left(\bar{x}, \bar{y}, S_{3}\right)=\frac{-i E_{0} e^{i x} \text { factor }}{m \lambda\left(S-S_{3}\right) R} \int_{\substack{\text { area }}} e^{-\frac{2 \pi i m}{\lambda\left(S-S_{3}\right)}\left[\bar{x}\left(x^{\prime}{ }_{0}+\ell_{1}\right)+\bar{y}\left(y^{\prime}{ }_{0}+\ell_{2}\right)\right]} d\left(x^{\prime}{ }_{0}+\ell_{1}\right) d\left(y^{\prime}{ }_{0}+\ell_{2}\right)$

which gives the light wave amplitude in the transverse plane at $S_{3}$. As before, the transmission function is taken as zero everywhere except at the pit location where it is unity. Since the integration is over $x_{0}^{\prime}$ and $y_{0}^{\prime}$, the expression in Equation 27 can be written as

$E\left(\bar{x}, \bar{y}, S_{3}\right)=\frac{-i E_{0} m e^{i} \times \text { factor }}{\lambda\left(S-S_{3}\right) R} e^{-\frac{2 \pi i m}{\lambda\left(S-S_{3}\right)}\left[\bar{x} \ell_{1}+\bar{y} \ell_{2}\right]} \iint e^{-\frac{2 \pi i m}{\lambda\left(S-S_{3}\right)}\left[\bar{x} x_{0}^{\prime}+\bar{y} y_{0}^{\prime}\right]} d x_{0}^{\prime} d^{\prime}{ }_{0}$

The off-centered pit location results in the phase factor

$\exp \left[\frac{-2 \pi \mathrm{im}}{\lambda\left(\mathrm{S}-\mathrm{S}_{3}\right)}\left(\overline{\mathrm{x}} \ell_{1}+\overline{\mathrm{y}} \ell_{2}\right)\right]$

appearing in Equation 28, but the double integral expression in Equation 28 is identical in form to the integral expression in Equation 17 evaluated earlier for a centered pit. The intensity in the plane at $S_{3}$ is found by multiplying $E\left(\bar{x}, \bar{y}, S_{3}\right)$ by its complex conjugate $E^{*}\left(\bar{x}, \bar{y}, S_{3}\right)$ which causes the phase factors to cancel in the expression

$I=E\left(\bar{x}, \bar{y}, S_{3}\right) E^{*}\left(\bar{x}, \bar{y}, S_{3}\right)$,

and hence the intensity in the plane at $S_{3}$ is independent of the pit position within the illuminated spot on the sphere. 
The foregoing analyses of the defect diffraction patterns can be summarized with the aid of Figure 7. When an isolated pit defect is illuminated with the system in Figure 3, the diffraction pattern observed in the transverse plane at $S_{3}$ will consist of a series of approximately circular, concentric rings, alternately bright and dark, centered about point $P$. An illuminated isolated scratch defect will produce a very narrow "line" diffraction pattern centered at point $\mathrm{P}$ and lying in the transverse plane at $S_{3}$. The orientation of the line diffraction pattern through $P$ will depend on the orientation of the scratch within the illuminated spot on the sphere. It is assumed that the beam diameter is sufficiently large, for a given focal length $F$ and sphere radius $R$, that the illuminated spot diameter is much larger (factor of 10 or 20 ) than the pit diameter or scratch width. Under these conditions the assumptions of infinite beam or lens aperture sizes are valid and the diffraction effects from the finite beam size are only $1 / 10$ or $1 / 20$ the size of the defect diffraction effects. Under these conditions the finite beam or aperture diffraction effects are de-coupled from and do not interfere with the defect diffraction effects.

In Figure 7 the central bright focused spot of light retroreflected from the defect-free portion of the surface within the illuminated spot is represented by the intense, off-scale peak centered at $P$. The total light energy in the defect diffraction pattern will be only $(1 / 10)^{2}$ to $(1 / 20)^{2}$ of the total specularly reflected light from the defect-free surface. Therefore, the bright focused spot will partially mask the central, relatively broad, maximum in the defect diffraction pattern. However, if the magnification of the optical arrangement is sufficiently large $(\geq 10)$, the first relative maximum in the defect diffraction pattern from defects approximately $25 \mu \mathrm{m}$ wide will occur "outside" the central bright focused spot of specular light. This is the case depicted in Figure 7. For smaller values of magnification the first relative maximum in the defect diffraction pattern will be nearer the point $P$ and hence will appear as "spikes" situated on the steep slopes of the intensity versus distance curve of the bright focused spot.

The symmetries exhibited by the isolated pit and scratch diffraction patterns in the transverse plane at $S_{3}$, and the stationary property of the patterns relative to point $P$ in this plane, make the geometry of the commercial photodetector array shown in Figures 2 and 3 appropriate for automated analyses of the defect diffraction pattern intensities. Accordingly, for approximately circular pits and for long narrow scratches the ring and wedge signals of the photodetector array centered at point $P$ in Figure 3 should be as shown in Figure 8. The signals provide a means of distinguishing between isolated pits and scratches and obtaining a measure of the defect sizes.

Alternate Photodetector Arrangement for Exposing Central Maximum of Defect Pattern and Minimizing Diffuse Light From "Rough" Superfinished Backgrounds

When the defect diffraction patterns are observed in the transverse plane at $S_{3}$ the central maximum of the pattern is partially masked by the very large bright focused spot of specularly reflected light, as discussed earlier. Also, unless the spherical surface background is very smooth (arithmetic average $\leq 0.5$ microinch) the diffuse reflected light appearing at the base of the bright focused spot can be of considerable magnitude and can mask the relative maxima in the defect diffraction 

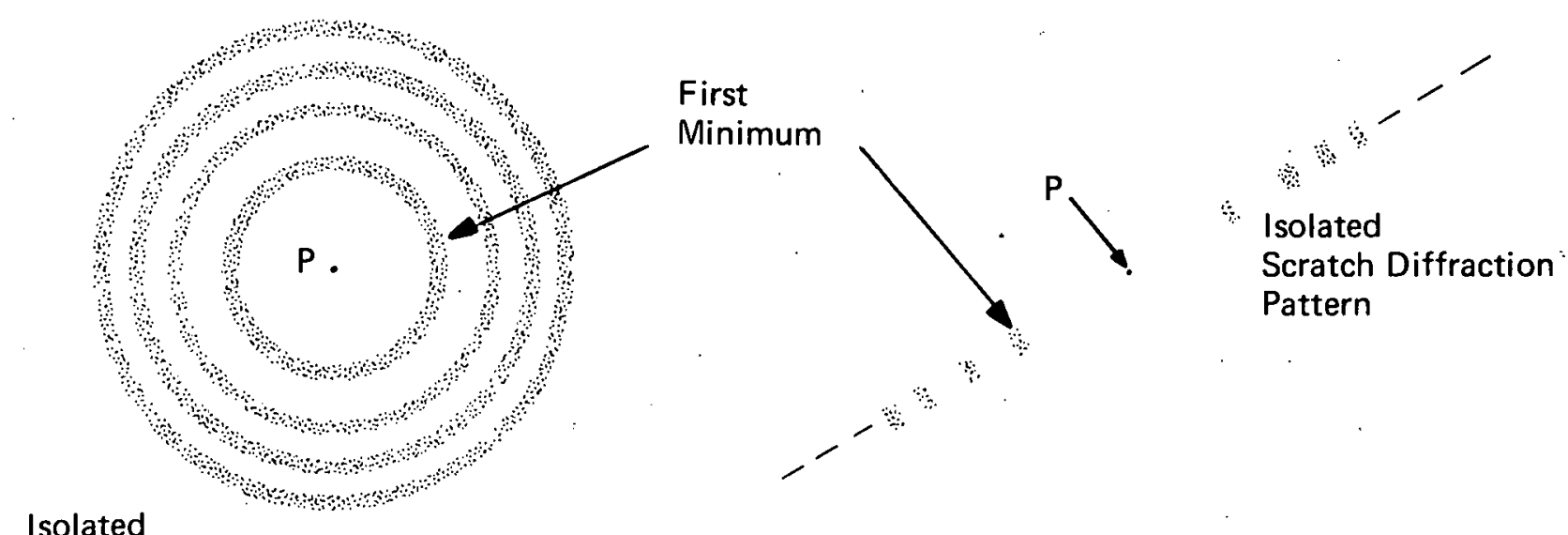

Isolated

Pit Diffraction

Pattern

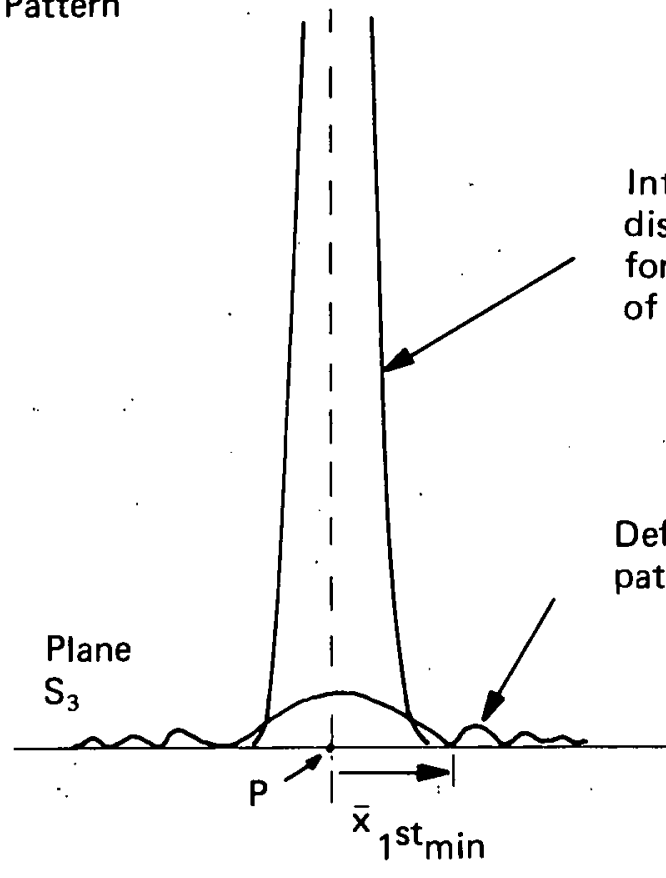

Intensity versus

distance from $P$

for focused spot

of specular light

Defect diffraction

pattern

Figure 7. Schematic Comparisons of Isolated Pit and Scratch Diffraction Pattern Intensities With Focused Spot of Specular Light Intensity in the Transverse Plane at $S_{3}$ in Figure 3 


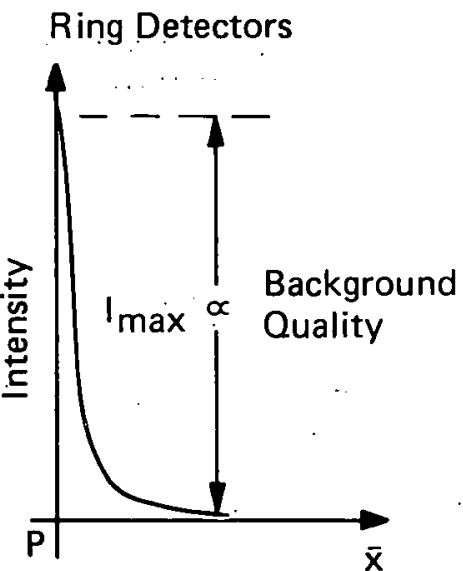

Wedge Detectors

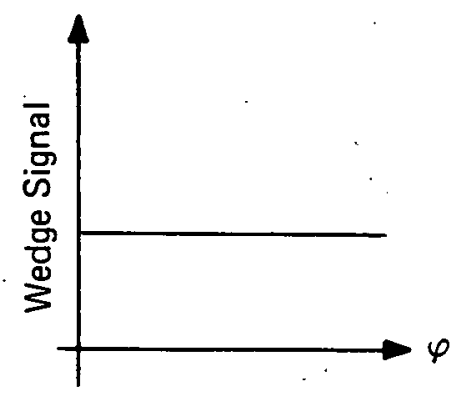

The Background Signal is symmetric because of randomness of the minute scratches and undulations.
Detector Signals
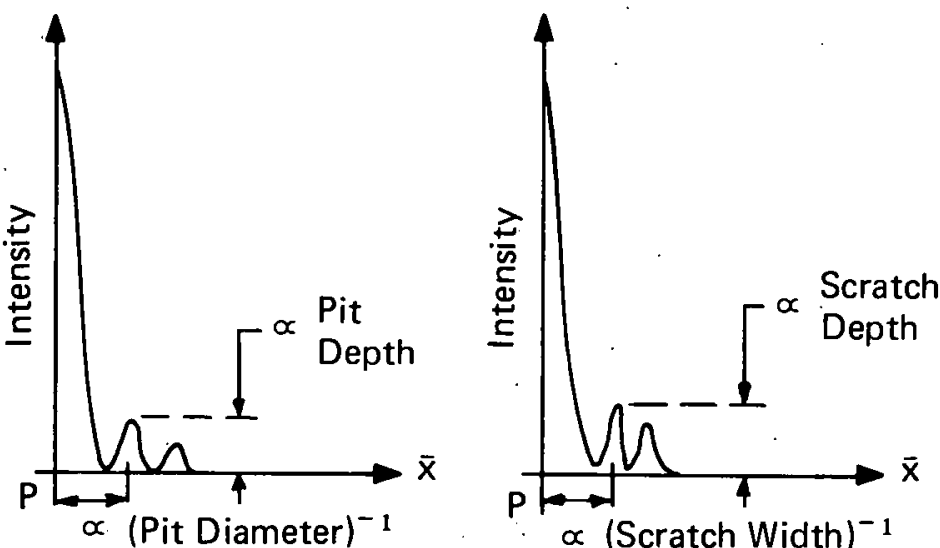

$\propto(\text { Scratch Width })^{-1}$

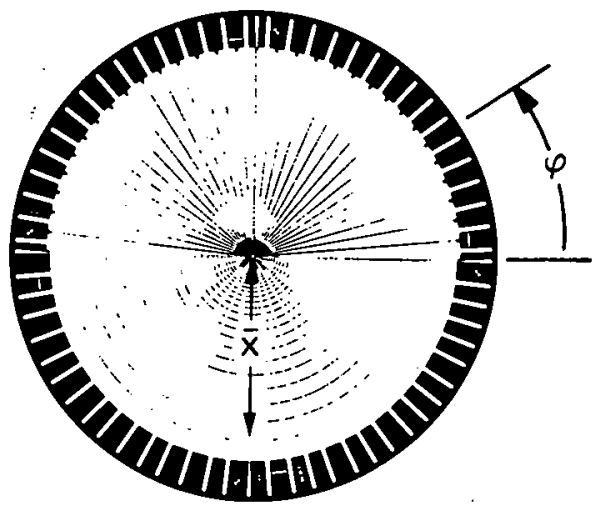

Commercial

Photodetector Array

$\bar{x}$ is the radial distance from center of detector. $\varphi$ is the angular coordinate measured about the

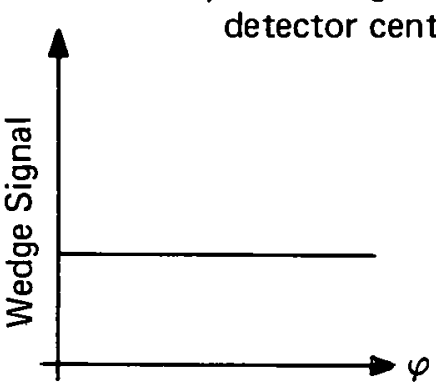

Isolated Pit Defect. A pit causes an alternately bright and dark ring diffraction pattern symmetric about center.

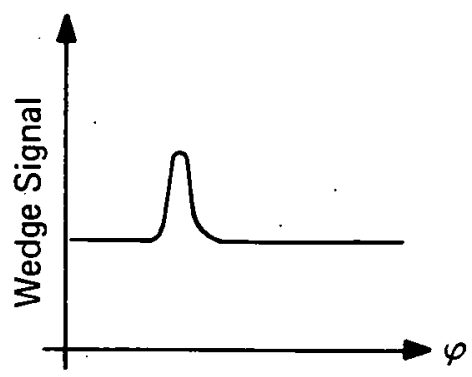

Isolated Scratch Defect. The angular position of wedge signal "Blip" depends on orientation of scratch on sphere.

Figure 8. Schematic Representations of Light Signals in Plane at $S_{3}$ for Isolated Pit and Isolated Scratch Defects 
pattern. These two masking effects can be overcome to some extent by sacrificing the stationary property of the pattern position about point $\mathrm{P}$ in Figure 3. That is, if the defect diffraction pattern is observed in a transverse plane at some position between the lens of focal length $F$ and the plane $S_{3}$ in Figure 3, then the entire central maximum of the pattern is visible because the specularly reflected light is not focused. Also, diffuse reflected light from a relatively "rough" superfinished background does not tend to significantly mask the higher order maxima in the pattern when the pattern is viewed in a plane just after the lens of focal length $F$. However, under these conditions the defect diffraction pattern is not in focus and its position in the plane of observation does depend on the defect location in the illuminated spot. Under these conditions the pit and scratch pattern symmetries do not match the ring and wedge detector symmetries of the commercial photodetector array. (In spite of this, earlier work ${ }^{2}$ showed that the commercial ring-wedge photodetector could be used to detect and distinguish among defect sizes for the case of off-centered and unfocused defect diffraction patterns.)

An alternate photodetector array which can be used to detect and distinguish defect diffraction patterns in a transverse plane just after the lens of focal length $F$ in Figure 3 is shown in Figure 9. The alternate photodetector array consists of a square array of 64 photodetectors, each of which would be connected with an amplifier of the commercial high speed data sampler associated with the 64-element commercial photodetector array. To achieve close-packing in space, the points of detection in the transverse plane can consist of one end of each of 64 light pipes; the opposite end of each pipe is then passed to a detector connected to the high speed data sampler. The signals from the detectors within the square array can be used to detect the presence of a defect diffraction pattern and can be analyzed to distinguish between a concentric ring pit pattern and a parallel line pattern from an isolated scratch. In this way the somewhat undesirable arbitrary position of a defect pattern in the plane of the square array of detectors is accounted for by detector signal analysis designed to first locate the center of the pattern and then, secondly, to analyze the intensity distribution from the center of the pattern outward, as is done with the commercial photodetector array for defect patterns centered at $\mathrm{P}$ in the plane at $\mathrm{S}_{\mathbf{3}}$ in Figure 3.

\section{Diffracted Light Intensity as a Function of Isolated Defect Depth}

\section{Theory}

When light is incident on a metal surface there is an interaction between the electromagnetic field of the light wave and induced fields at the metal surface. To treat the electromagnetic interaction exactly, the boundary conditions for Maxwell's electromagnetic field equations must be satisfied. This is a formidable task if surface roughness is to be taken into account. Fresnel's equations, which relate incident, refracted, and reflected intensities for light incident at an air-dielectric interface, provide an explanation for the simple laws of reflection and refraction; however, Fresnel's equations presuppose a perfectly plane interface free from any residual roughness. ${ }^{5}$ Davies developed a theory based on the Kirchoff boundary conditions, which are somewhat less accurate than the Maxwell boundary conditions, that describes reflected light intensities by considering the 


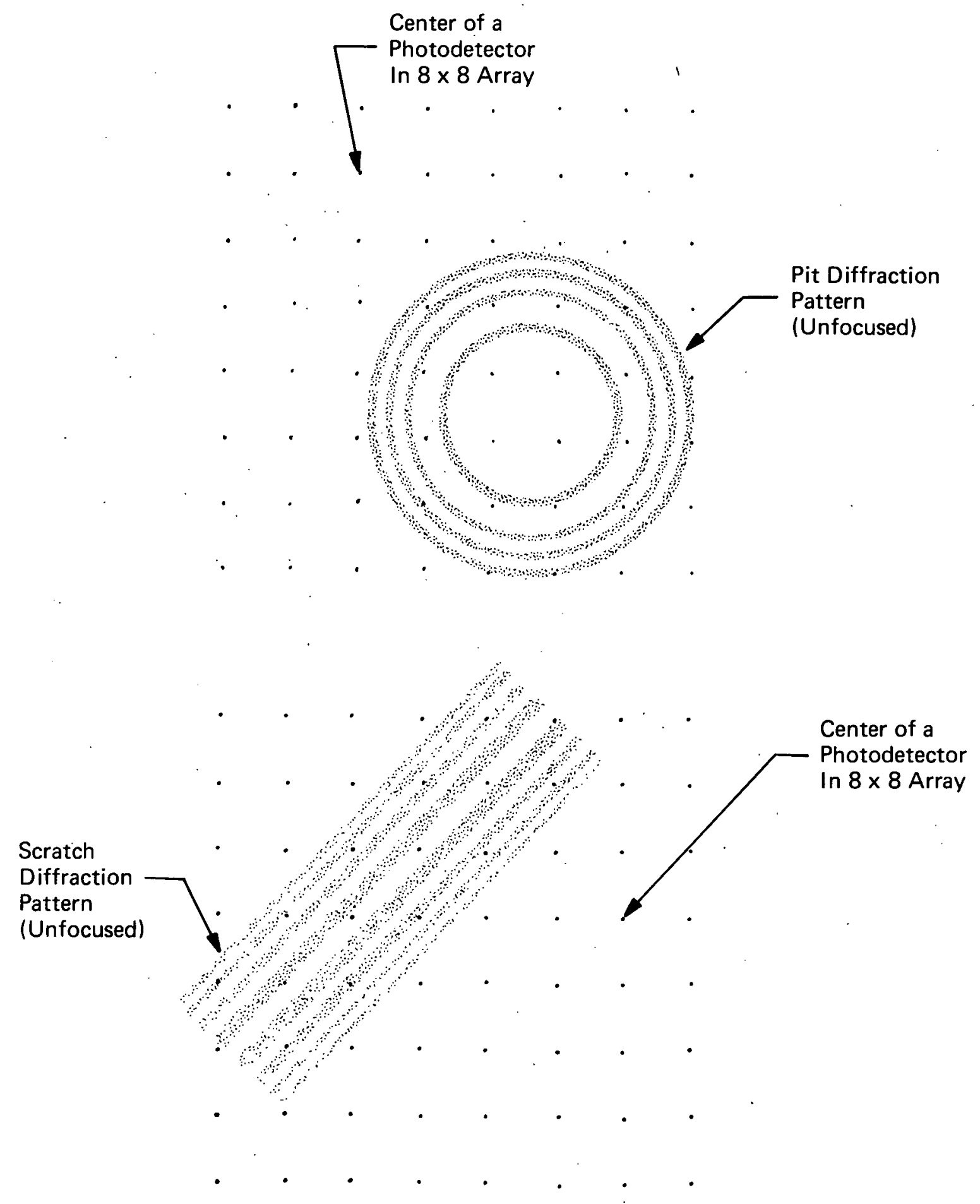

Figure 9. Possible Photodetector Array for Analyzing Defect Diffraction Patterns in a Transverse Plane Between the $S_{3}$ Plane and the Lens of Focal Length $F$ in Figure 3 
statistical nature of surface roughness. ${ }^{6}$ Davies' theory and treatments of Porteus based on it adequately describe and correlate reflected light intensities with the average surface roughness or "background."7

Elson and Richtie developed a theory which satisfies the Maxwell boundary conditions and gives the light intensities reflected from a rough surface in terms of the roughness profile of the surface. ${ }^{8}$ For light incident normally on a surface, Elson and Richtie showed that the differential light energy reflected into a differential solid angle in a direction specified by the angular coordinates $(\theta, \varphi)$ is

$\frac{d E}{d \Omega}=\frac{d_{s}}{d \Omega}+\frac{d E_{\rho}}{d \Omega}$

where the s- polarized light is

$\frac{\mathrm{dE}_{\mathrm{s}}}{\mathrm{d} \Omega}=\frac{(2 \pi)^{4}}{\lambda^{4} \pi^{2} \mathrm{~L}_{0}^{2}} \cos ^{2} \theta \sin ^{2} \varphi|\mathrm{A}|^{2}$,

$\mathrm{L}_{0}^{2}$ is the illuminated spot area; $\lambda$ is the wavelength, and

$A=\int_{\vec{r}} \xi(\vec{r}) e^{-i \vec{k} \cdot \vec{r}} d \vec{r}$

In the latter, $\vec{k}$ is the wave vector $(|\vec{k}|=2 \pi / \lambda)$ of the scattered light into the direction $(\theta, \varphi), \vec{r}$ is a two-dimensional vector parallel to the average planar surface, and $\xi(\vec{r})$ is the surface roughness function specifying the surface topology. The p-polarized light is given by

$\frac{d E_{p}}{d \Omega}=\frac{(2 \pi)^{4}}{\lambda^{4} \pi^{2} L_{0}^{2}} \cos ^{2} \theta \cos ^{2} \varphi\left[\frac{\sin ^{2} \theta-\epsilon_{1}}{\sin ^{2} \theta-\epsilon_{1} \cos ^{2} \theta}\right]|A|^{2}$,

where $\epsilon_{1}$ is the dielectric permittivity of the medium on which the light is incident.

For angles of observation $\theta$ which are relatively small, the $\sin \theta$ factor can be neglected and $\cos \theta \operatorname{set}$ equal to one, in which case the total light reflected into the $(\theta, \varphi)$ direction is, from Equation 29 ,

$\frac{\mathrm{dE}}{\mathrm{d} \Omega}=\frac{(2 \pi)^{4}}{\lambda^{4} \pi^{2} \mathrm{~L}_{0}^{2}}\left|\int_{\overrightarrow{\mathrm{r}}} \xi(\overrightarrow{\mathrm{r}}) \mathrm{e}^{-\mathrm{i} \overrightarrow{\mathrm{k}} \cdot \overrightarrow{\mathrm{r}}} \mathrm{d} \overrightarrow{\mathrm{r}}\right|^{2}$

Equation 33 can be used to calculate the amount of energy diffracted into space at small angles off normal when light is incident normally on a surface characterized by a roughness profile $\xi(\overrightarrow{\mathrm{r}})$. 


\section{Isolated Scratch Defect}

The integral in Equation 31 can be evaluated for an isolated scratch defect* by referring to Figure 10. It is assumed that the scratch of width $w$ at the surface level and depth $h$ is located on a surface background which is characterized by an arithmetic average much smaller than the depth of the scratch. The scratch can thus be assumed to exist on an ideally smooth surface, and the function $\xi(\vec{r})$ is defined by

$\xi=0 \quad x<-w / 2 ; x>w / 2$

$\xi=-\mathrm{h}\left(1+\frac{2 \mathrm{x}}{\mathrm{w}}\right)-\frac{\mathrm{w}}{2} \leq \mathrm{x} \leq 0$

$\xi=-\mathrm{h}\left(1-\frac{2 \mathrm{x}}{\mathrm{w}}\right) 0 \leq \mathrm{x} \leq \frac{\mathrm{w}}{2}$

If $L$ is the length of the scratch, then

$$
\begin{aligned}
& A=\int_{\vec{r}} \xi(r) e^{-i \vec{k} \cdot \vec{r}} d \vec{r} \\
& =-h \int_{-\frac{L}{2}}^{\frac{L}{2}} \int_{-\frac{w}{2}}^{0}\left(1+\frac{2 x}{w}\right) e^{-i k_{x} x} e^{-i k_{y} y} d x d y \\
& -h \int_{-\frac{L}{2}}^{\frac{L}{2}} \int_{0}^{\frac{w}{2}}\left(1-\frac{2 x}{w}\right) e^{-i k_{x} x} e^{-i k_{y} y} d x d y .
\end{aligned}
$$

It is shown in Appendix D that the integrals in Equation 35 yield

$$
\frac{\mathrm{dE}}{\mathrm{d} \Omega}=\frac{(2 \pi)^{4}}{\lambda^{4} \pi^{2} \mathrm{~L}_{0}^{2}} \mathrm{~h}^{2} \frac{\left[\cos \left(\frac{\pi \mathrm{w}}{\lambda} \sin \theta\right)-1\right]^{2}}{\mathrm{w}^{2}\left(\frac{2 \pi}{\lambda} \sin \theta\right)^{4}} \text {, }
$$

${ }^{*}$ Private communication with J.M. Elson. ${ }^{8}$ 

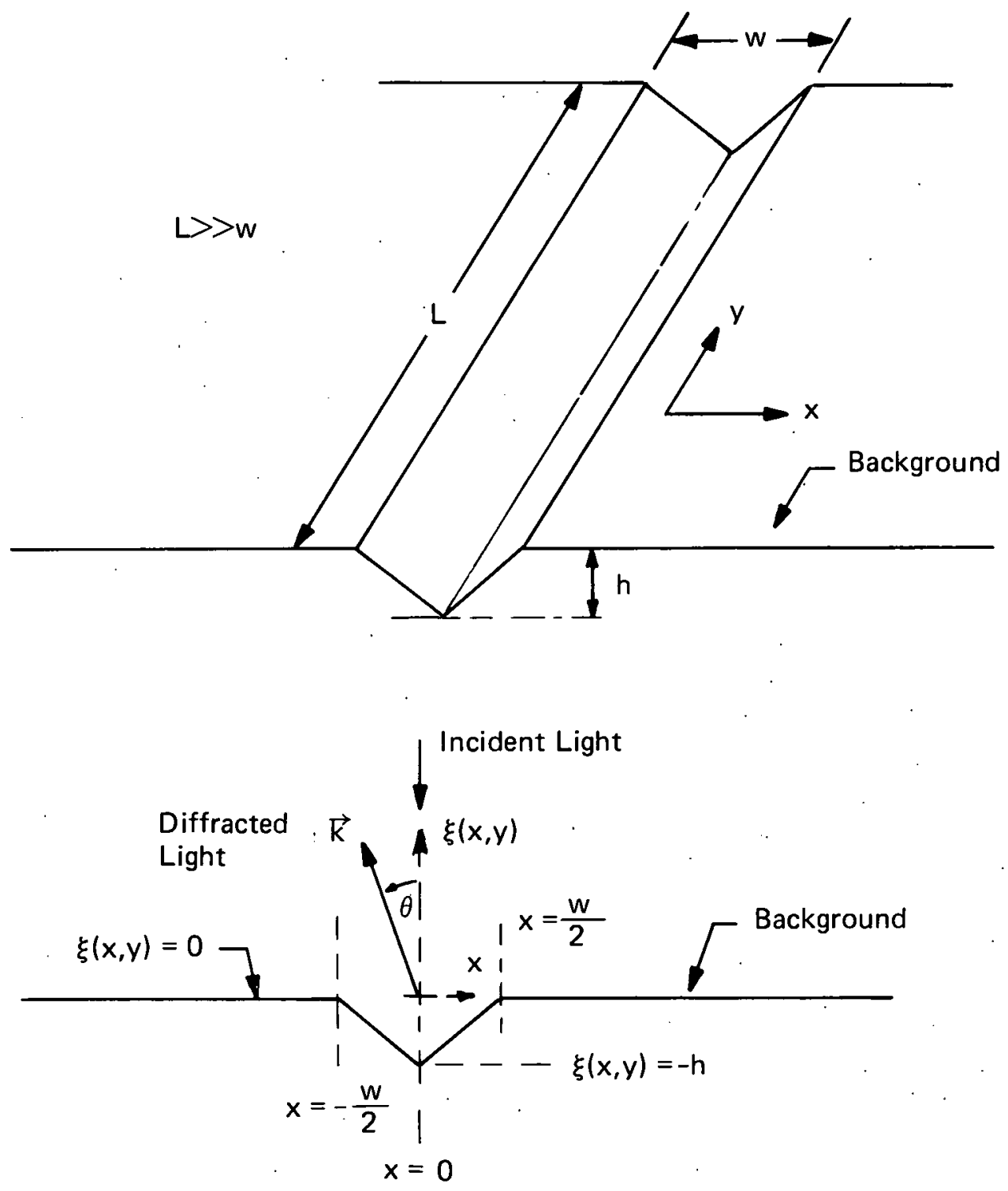

$$
\begin{aligned}
& \xi=0 \quad x<-\frac{w}{2}, x>\frac{w}{2} \\
& \xi=-h\left(1+\frac{2 x}{w}\right) \quad-\frac{w}{2} \leq x \leq 0 \\
& \xi=-h\left(1-\frac{2 x}{w}\right) \quad 0 \leq x \leq \frac{w}{2}
\end{aligned}
$$

Figure 10. Geometry Used in Determining Diffraction Pattern Intensity Distribution From an Isolated Scratch Defect on a Superfinished Background 
when the scratch length $L$ is very long compared with the width or depth. According to Equation 36 the light energy diffracted at small angles relative to the normal is proportional to the square of the scratch depth $h$ for constant scratch width $w$. For a constant depth scratch, the maxima in the diffracted light occur at angles $\theta$ varying inversely with the scratch width $\mathrm{w}$. The minima occur at angles $\theta$ so that $\cos \left(\frac{\pi \mathrm{w}}{\lambda} \sin \theta\right)-1=0$ or $\theta=\operatorname{arc} \sin \left[2 \frac{\lambda}{\mathrm{w}} \mathrm{N}\right]$ where $\mathrm{N}=1,2,3, \cdots$. Therefore, according to this theory the minima occur at twice the distances from the center predicted by Fraunhofer theory, leading to Equation 5a.

\section{Isolated Pit Defect}

For an isolated pit defect of radius $\mathrm{R}$ and depth $\mathrm{h}$ present on an ideally smooth background surface, the surface profile function $\xi(r)$ in Equations 31 and 33 takes the form

$\xi=0$ for $|x|,|y|>R$

$\xi=-h\left(1-\frac{r}{R}\right)$ for $|x|,|y| \leq R$

where the pit is assumed to be a symmetrical cone-shaped defect. The factor $\vec{k} \cdot \vec{r}$ in Equation 31 can be written

$\vec{k} \cdot \vec{r}=k_{x} x+k_{y} y$

$=\mathrm{kr} \sin \theta \cos (\alpha-\varphi)$

where the angles $\theta, \varphi$, and $\alpha$ are defined by the geometry in Figure 11. The integral in Equation 31 can be written

$A=-h \int_{0}^{R} \int_{0}^{2 \pi}\left(1-\frac{r}{R}\right) r e^{-i k r \sin \theta \cos (\alpha-\varphi)} d \alpha d r$

Without loss of generality, the angle $\varphi$ can be taken as zero because the pit is symmetric about the vertical $\mathrm{z}$ axis. Equation 39 thus becomes

$A=-h \int_{0}^{R} \int_{0}^{2 \pi}\left(i-\frac{r}{R}\right) r e^{-i k r \sin \theta \cos \alpha} d \alpha d r$

The use of a Bessel function identity allows Equation 40 to be put into the form 

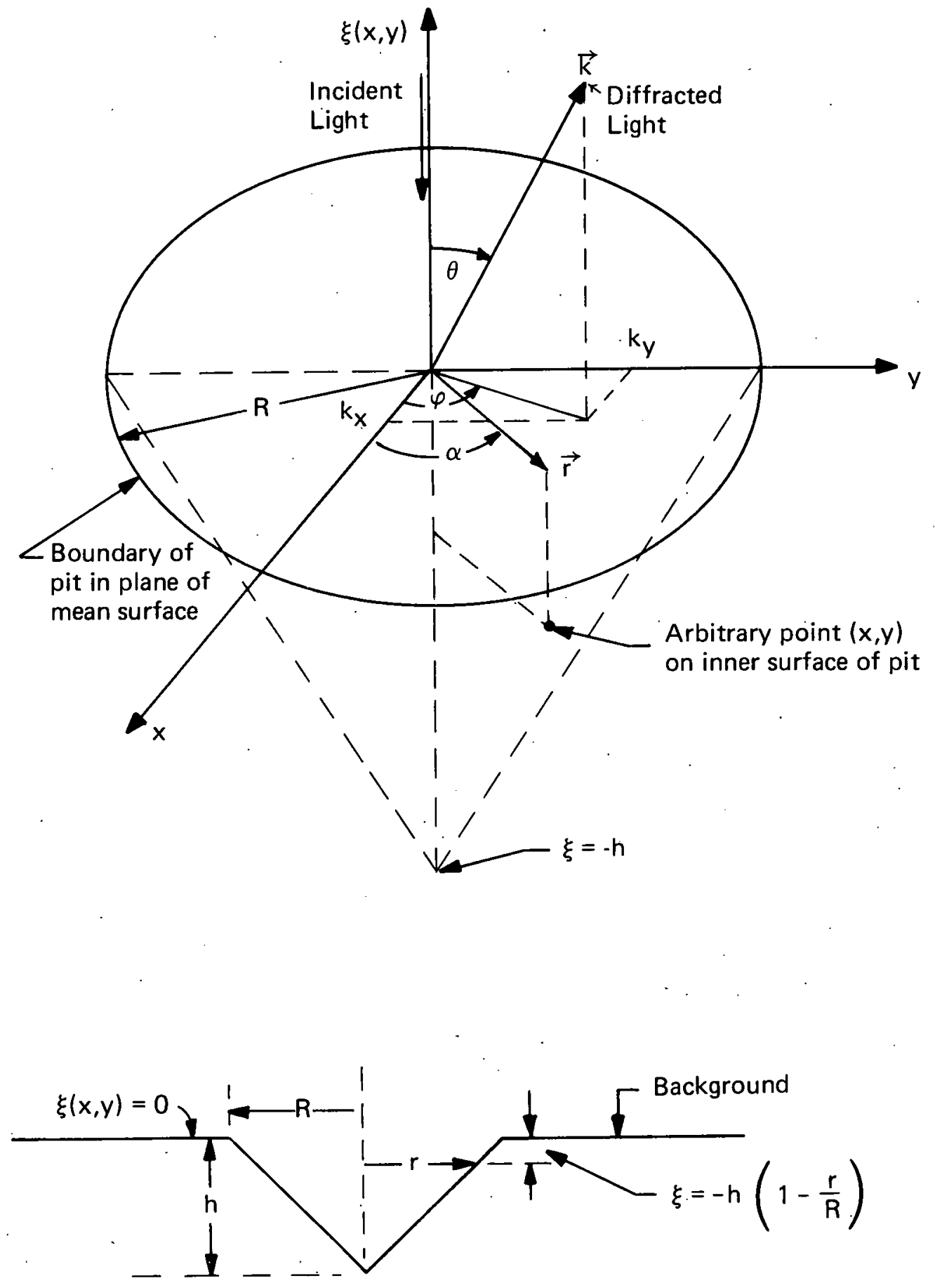

Figure 11. Geometry Used in Determining Diffracted Light Intensity Distribution From an Isolated Pit of Diameter 2R and Depth $\mathrm{h}$ Present on Superfinished Background 
$A=-\frac{h}{2 \pi} \int_{0}^{R}\left(1-\frac{r}{R}\right) r J_{0}(k r \sin \theta) d r$

where $\mathrm{J}_{0}$ is the zero order Bessel function. When the Bessel function is expanded, term-by-term integration is carried out, and the resulting terms are recombined, the following expression is obtained (see Appendix E).

$A=-\frac{h R^{2}}{2 \pi} \sum_{\ell=0}^{\infty} \frac{(-1)^{\ell}(k R \sin \theta)^{2 \ell}}{2^{2 \ell}(\ell !)^{2}(2 \ell+2)(2 \ell+3)}$

The diffracted light, at small angles off normal, is given by Equations 33 and 42

$\frac{\mathrm{dE}}{\mathrm{d} \Omega}=\frac{(2 \pi)^{4}}{\lambda^{4} \pi^{2} \mathrm{~L}_{0}^{2}} \frac{\mathrm{h}^{2} \mathrm{R}^{4}}{4 \pi^{2}}\left\{\left.\sum_{\ell=0}^{\infty} \frac{(-1)^{\ell}(\mathrm{kR} \sin \theta)^{2 \ell}}{2^{2 \ell}(\ell !)^{2}(2 \ell+2)(2 \ell+3)}\right|^{2}\right.$

where $\mathrm{k}=2 \pi \div$ wavelength. For small angles, the sine function can be approximated by the tangent and written

$\sin \theta \approx \tan \theta=y / L$

where $\mathrm{L}$ is the distance of observation from the illuminated pit and $\mathrm{y}$ is the distance measured in a transverse plane at distance $\mathrm{L}$ from the pit. It is thus seen that the light diffracted into the off-normal direction is proportional to the square of the pit depth $h$, for a given pit diameter. The distance to the first minimum in the pattern can be calculated from Equation 43. This was done for a distance $\mathrm{L}=254 \mathrm{~mm}$, wavelength $\lambda=632.8 \mathrm{~nm}$, and pit diameters 25,51 , and $102 \mu \mathrm{m}$. The results are shown in Figure 12 and are within 35 percent of the first minima calculated from Fraunhofer theory.

In the previous case, the isolated pit defect was treated as an "inverted" cone with its axis perpendicular to the mean surface level and its circular base of diameter $2 \mathrm{R}$ lying in the plane of the mean surface. The apex of the cone, situated at a distance $h$ directly below the mean surface, represents the maximum pit depth.

Consider next the model of an isolated pit defect shown in Figure 13. The base of the truncated cone of radius $\mathrm{R}_{4}$ lies in the plane of the mean surface and corresponds to a circular pit of diameter $2 R_{4}$. The cone angle varies in a step function with depth as indicated, but the pit possesses symmetry about its axis in any plane parallel to the mean surface. The functions $\xi(r)$ for the depth of the pit as a function of the pit cross-sectional radius are given below. 


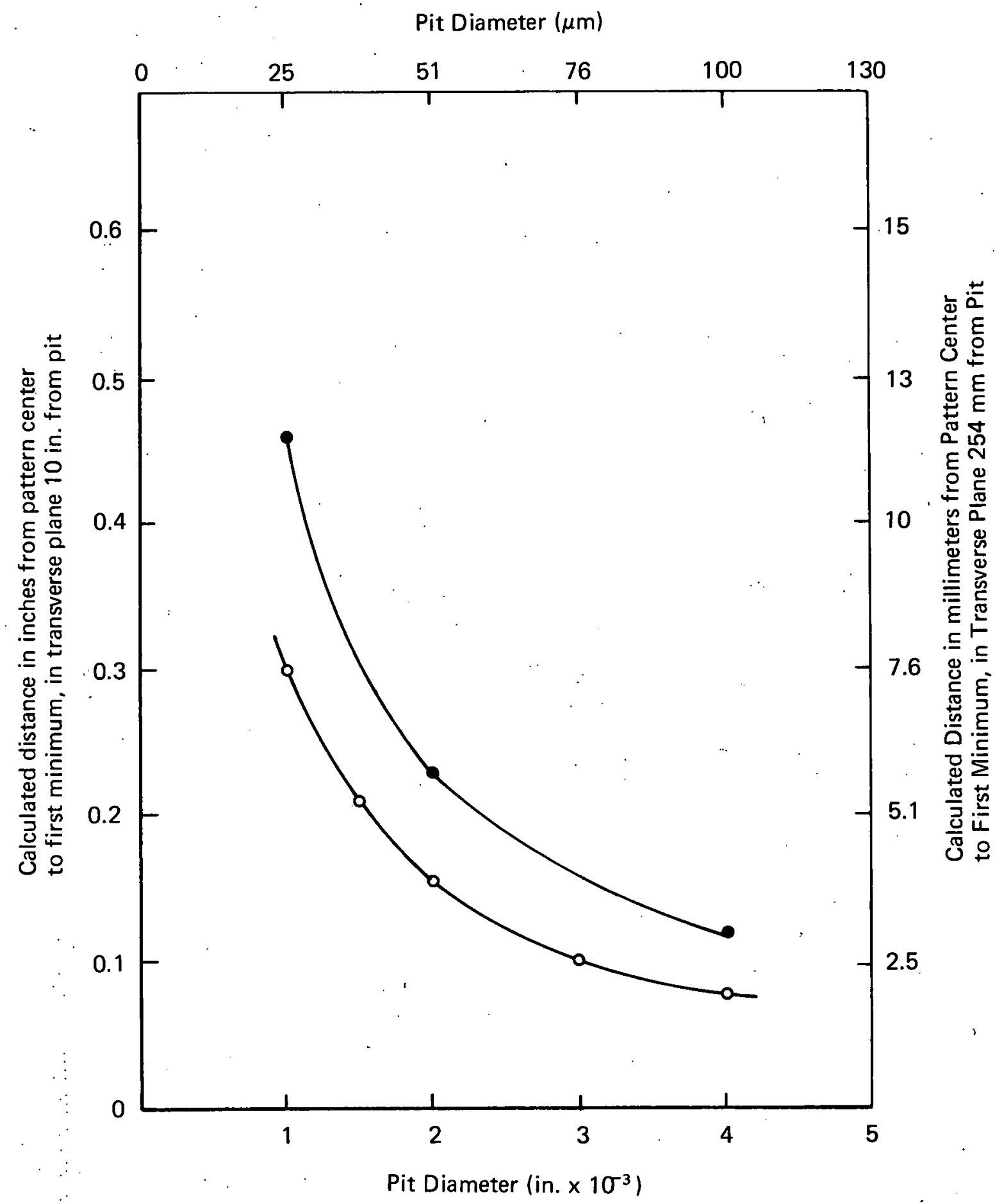

Figure 12. Comparison of Calculated Position of Pit Diffraction Pattern First Minimum Based on Diffracted Light From Model Pit in Figure 11, and Fraunhofer Diffraction by a Circular Aperture of Diameter Equal to the Pit Diameter 


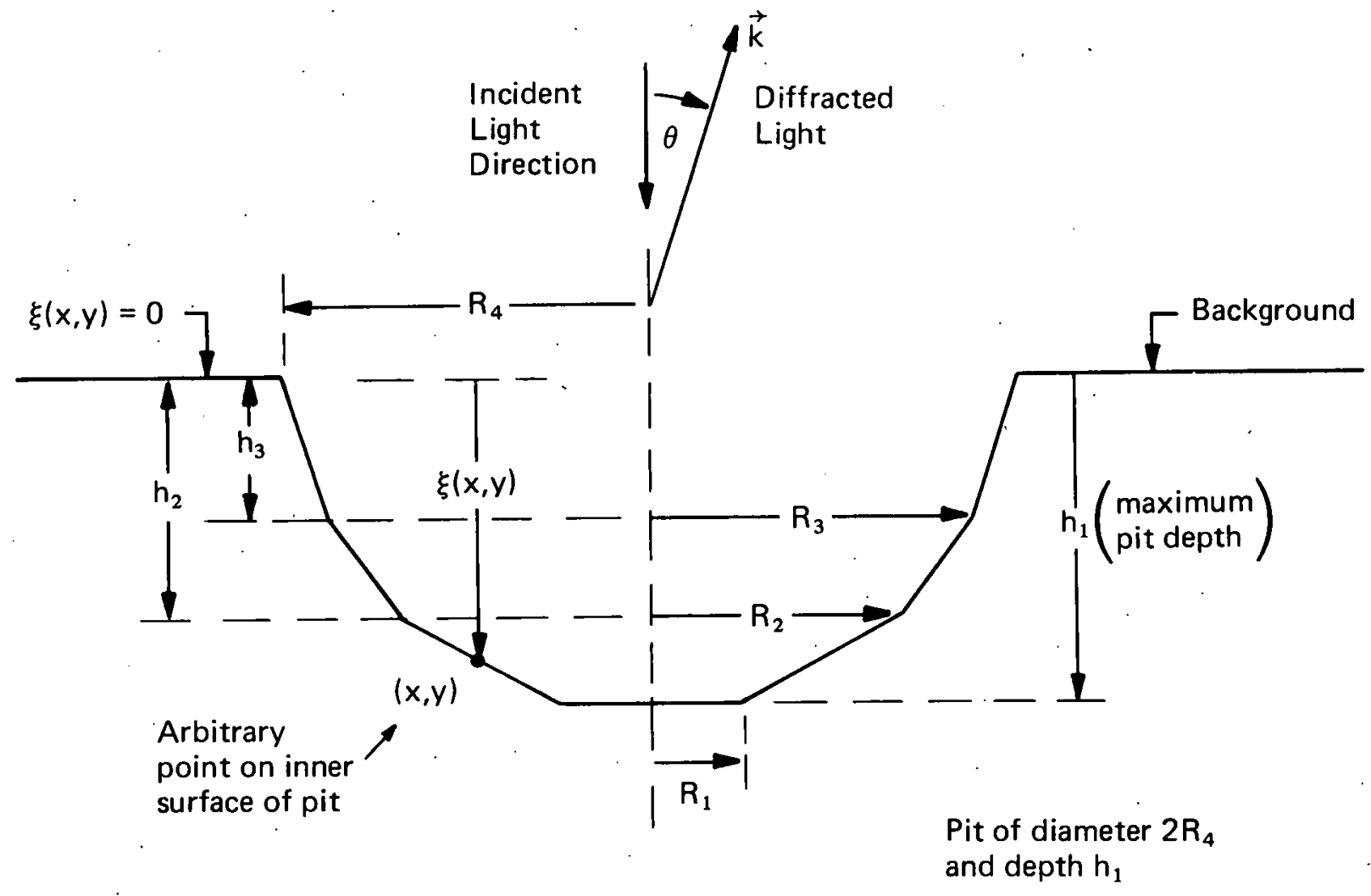

Figure 13. Cross-Section of a Model Pit Having Sides Which Are Portions of Cones of Different Slopes (The pit is symmetric about an axis perpendicular to the background mean surface.) 


$$
\begin{array}{ll}
\xi_{1,2}(r)=-\frac{h_{1} R_{2}-h_{2} R_{1}}{R_{2}-R_{1}}\left[1-\frac{h_{1}-h_{2}}{h_{1} R_{2}-h_{2} R_{1}} r\right] & R_{1} \leq r \leq R_{2} \\
\xi_{2,3}(r)=-\frac{h_{2} R_{3}-h_{3} R_{2}}{R_{3}-R_{2}}\left[1-\frac{h_{2}-h_{3}}{h_{2} R_{3}-h_{3} R_{2}} r\right] & R_{2} \leq r \leq R_{3} \\
\xi_{3,4}(r)=-\frac{h_{3} R_{4}}{R_{4}-R_{3}}\left(1-\frac{r}{R_{4}}\right) & R_{3} \leq r \leq R_{4}
\end{array}
$$

To determine the diffraction pattern intensity distribution resulting when the model pit of Figure 13 is illuminated at normal incidence, it is necessary to evaluate the integral

$$
A=\int_{\vec{r}} \xi(r) e^{-i \vec{k} \cdot \vec{r}} d \vec{r}
$$

appearing in Equation 31 for each of the three regions of $r$ in Equation 44. The integral forms are given by

$$
A_{\text {total }}=\int_{\vec{r}} \xi_{1,2}(r) e^{-i \vec{k} \cdot \vec{r}} d \vec{r}+\int_{\vec{r}} \xi_{2,3}(r) e^{-i \vec{k} \cdot \vec{r}} d \vec{r}+\int_{\vec{r}} \xi_{3,4}(r) e^{-i \vec{k} \cdot \vec{r}} d \vec{r}
$$

where $\xi_{1,2}(r), \xi_{2,3}(r)$, and $\xi_{3,4}(r)$ are expressed in Equation 44. The integral over $\xi_{1,2}(r)$ gives the amplitude of light diffracted into small angles off the surface normal by that portion of the pit in Figure 13 lying between the two parallel planes at distances $h_{1}$ and $h_{2}$ below the mean surface level. This portion of the pit varies in radius from the value $R_{1}$ to $R_{2}$. The integral over $\xi_{2,3}(r)$ gives the light amplitude diffracted by the portion of the pit varying in radius from $R_{2}$ to $R_{3}$ and lying between the two parallel planes at distances $h_{2}$ and $h_{3}$ below the surface. Finally, the integral over $\xi_{3,4}(r)$ represents the light diffracted by that portion of the pit extending from the surface level down to the parallel plane at depth $h_{3}$.

References to Figure 13 and Equations 38, 40, and 44 show that the first integral in Equation 45 can be put in the form

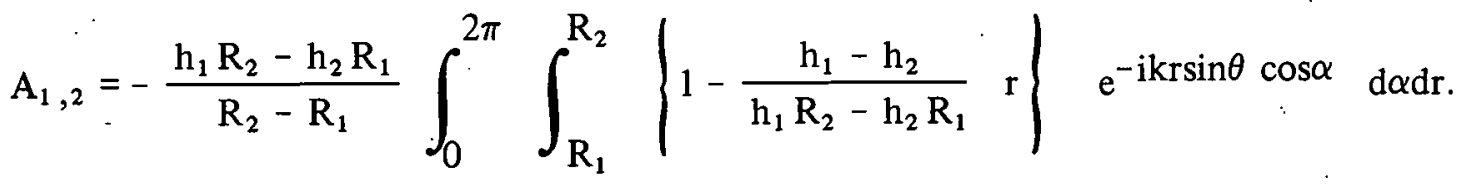

In Appendix $\mathrm{F}$ it is shown that 


$$
\begin{aligned}
& A_{1,2}=-\frac{h_{1} R_{2}-h_{2} R_{1}}{2 \pi\left(R_{2}-R_{1}\right)} \cdot\left[R_{2}^{2} \sum_{\ell=0}^{\infty} \frac{(-1)^{\ell}\left(k R_{2} \sin \theta\right)^{2 \ell}}{2^{2 \ell}(\ell !)^{2}} \cdot \frac{2 \ell\left(1-R_{2} h_{12}\right)+3-2 R_{2} h_{12}}{(2 \ell+2)(2 \ell+3)}\right. \\
& \left.+R_{1}^{2} \sum_{\ell=0}^{\infty} \frac{(-1)^{\ell}\left(k R_{1} \sin \theta\right)^{2 \ell}}{2^{2 \ell}(\ell !)^{2}} \cdot \frac{2 \ell\left(1-R_{1} h_{12}\right)+2 R_{1} h_{12}-3}{(2 \ell+2)(2 \ell+3)}\right]
\end{aligned}
$$

where $h_{12} \equiv\left(h_{1}-h_{2}\right) /\left(h_{1} R_{2}-h_{2} R_{1}\right)$. From the form of the expressions in Equation 44 it can be seen that the integral over $\xi_{2,3}(r)$ is identical in mathematical form to that of Equation 46 except that the subscripts 1 and 2 are replaced by 2 and 3, respectively. Likewise, the third integral in Equation 45 yields an expression of the form of Equation 47 with the subscripts 1 and 2 replaced by 3 and 4 , respectively. In the latter, $h_{4}$ is identically zero because this corresponds to the "top" of the pit at the mean surface level in Figure 13.

When Equation 47 and the two additional equations obtained by permuting the indices as described above are combined to provide an expression for the total light amplitude diffracted at the angle $\theta$, the diffraction pattern intensity is then determined by Equation 33. The following expression for the total diffracted light amplitude is derived in Appendix F.

$$
\begin{aligned}
& \frac{d E}{d \Omega}=\left[A_{1,2}+A_{2,3}+A_{3,4}\right]^{2} \frac{(2 \pi)^{4}}{\lambda^{4} \pi^{2} L_{0}^{2}} \\
& =\frac{(2 \pi)^{4}}{\lambda^{4} \pi^{2} L_{0}^{2}}\left\{\sum_{i=1}^{3} \frac{H_{i, i+1}}{2 \pi} \sum_{\ell=0}^{\infty} \frac{(-1)^{\ell}(k \sin \theta)^{2 \ell}}{2^{2 \ell}(\ell !)^{2}(2 \ell+2)(2 \ell+3)}\right. \\
& \left.\times\left[R_{i+1}^{2 \ell+2}\left(2 \ell\left(1-R_{i+1} h_{i, i+1}\right)+3-2 R_{i+1} h_{i, i+1}\right)+R_{i}^{2}(\ell+1)\left(2 \ell\left(R_{i} h_{i, i+1}-1\right)-3+2 R_{i} h_{i, i+1}\right)\right]\right\}^{2}, \\
& \text { where } h_{i, i+1}=\frac{\left(h_{i}-h_{i+1}\right)}{h_{i} R_{i+1}-h_{i+1} R_{i}} \text { and } H_{i, i+1}=-\frac{\left(h_{i} R_{i+1}-i h_{i+1} R_{i}\right)}{R_{i+1}-R_{i}}
\end{aligned}
$$

In the latter equation $\mathrm{k}$ is $2 \pi / \lambda$, which has the value $2.522 \times 10^{5} \mathrm{in}^{-1}\left(9.929 \mu \mathrm{m}^{-1}\right)$ for the helium neon laser wavelength $\lambda=632.8 \mathrm{~nm}$. For pit radii and depths in the range typically encountered on superfinished surfaces, the calculated intensities indicate that the series extending from $\ell=0$ to $\infty$ converges very rapidly for that part of the diffraction pattern extending from the center outward to the first minimum, first relative maximum, and the second minimum. The terms of the series alternate in sign because of the $(-1)^{\ell}$ factor, but the pit dimensions involved are such that the calculated intensity changes only by an amount less than one part per million for $\ell=10$. Therefore, for practical purposes the series can be said to converge within the first 10 terms. 
Equation 48 was used to calculate the diffraction pattern intensity distribution in a plane $254 \mathrm{~mm}$. distant from and parallel to the mean surface level for the case of an illuminated pit with radius $\mathrm{R}_{4}=25 \mu \mathrm{m}$ and a maximum depth $\mathrm{h}_{1}$ of $5 \mu \mathrm{m}$. With $\mathrm{R}_{4}$ and $\mathrm{h}_{1}$ fixed at these values, the pit radius $R_{3}$ was varied from 0.0003 to 0.0008 inch $(7.6 \mu \mathrm{m}$ to $20.3 \mu \mathrm{m})$ in the plane parallel to the surface at a distance 66.7 microinches $(1.67 \mu \mathrm{m})$ below the surface; in the plane at distance $h_{2}=133.3$ microinches $(3.33 \mu \mathrm{m})$ below the surface the pit radius $R_{2}$ was varied from 0.0001 to 0.00055 inch $(2.5$ to $13.8 \mu \mathrm{m})$. The corresponding value of $\mathrm{z}$, in the transverse plane $254 \mathrm{~mm}$ distant from the surface, at which the first minimum in the pattern occurred varied by only 8 percent from the first minimum position in the case of a pit of the kind shown in Figure 11 with a value of $R$ equal to the value of $R_{4}$ in the present case. That is, the first minimum occurs at a value of $z=0.23$ inch $(5.8 \mathrm{~mm})$ for a pit of radius $\mathrm{R}=0.001$ inch $(25 \mu \mathrm{m})$ and of the type shown in Figure 11 .

In the present case of a constant pit radius $R_{4}=0.001$ inch, the first minimum position varied from only 0.23 to 0.25 inch although the radius $R_{2}$ was varied by 550 percent and the radius $R_{3}$ by 267 percent. It foliows that the first minimum in the diffraction pattern is relatively insensitive to the values of $R_{2}$ and $R_{3}$ and is dependent primarily on the pit radius $R_{4}$ at the mean surface level.

Calculations were also made with Equation 48 to determine the dependence of the diffraction pattern intensity on pit depth for a constant pit diameter of $2 \mathrm{R}_{4}=0.0002$ inch $(50 \mu \mathrm{m})$. The maximum pit depth $h_{1}$ was varied from 20 to 200 microinches $(0.5$ to $5 \mu \mathrm{m})$ and the intermediate pit depths $h_{2}$ and $h_{3}$ were arbitrarily taken as 85.7 percent and 57.1 percent, respectively, of the corresponding maximum depth $h_{1}$. A plot of the calculated first relative maximum in the intensity pattern versus pit maximum depth is shown in Figure 14. The intensity is nearly proportional to the square of the depth in the depth range 0 to 200 microinches as seen by the comparison between the calculated data and the best-fit curve of the form Intensity $=\mathrm{Ah}_{1}^{2}$, where $\mathrm{A}=2.2 \times 1 \sigma^{-4} \mu \mathrm{in}^{-2}$ $\left(0.34 \mu \mathrm{m}^{-2}\right)$. The maximum range in defect depth, for a given wavelength, over which the approximations of Reference 8 and the subsequent Equations 36, 43, and 48 are valid is not known.

\section{ACCOMPLISHMENTS}

When an isolated defect such as a pit or scratch on a highly reflective metal sphere is illuminated with laser light, a characteristic diffraction pattern is observed in the reflected light. An optical system was designed for conveniently displaying the diffraction pattern on a photodetector array for rapid, automated analysis of the pattern intensity distribution. Theoretical expressions were derived which relate the diffraction pattern dimensions and intensities to the defect size and the optical system geometry. Experimental confirmation of the theory was obtained for the isolated pit diffraction pattern size and for the invariance of the defect pattern position relative to the defect position in the illuminated spot. 


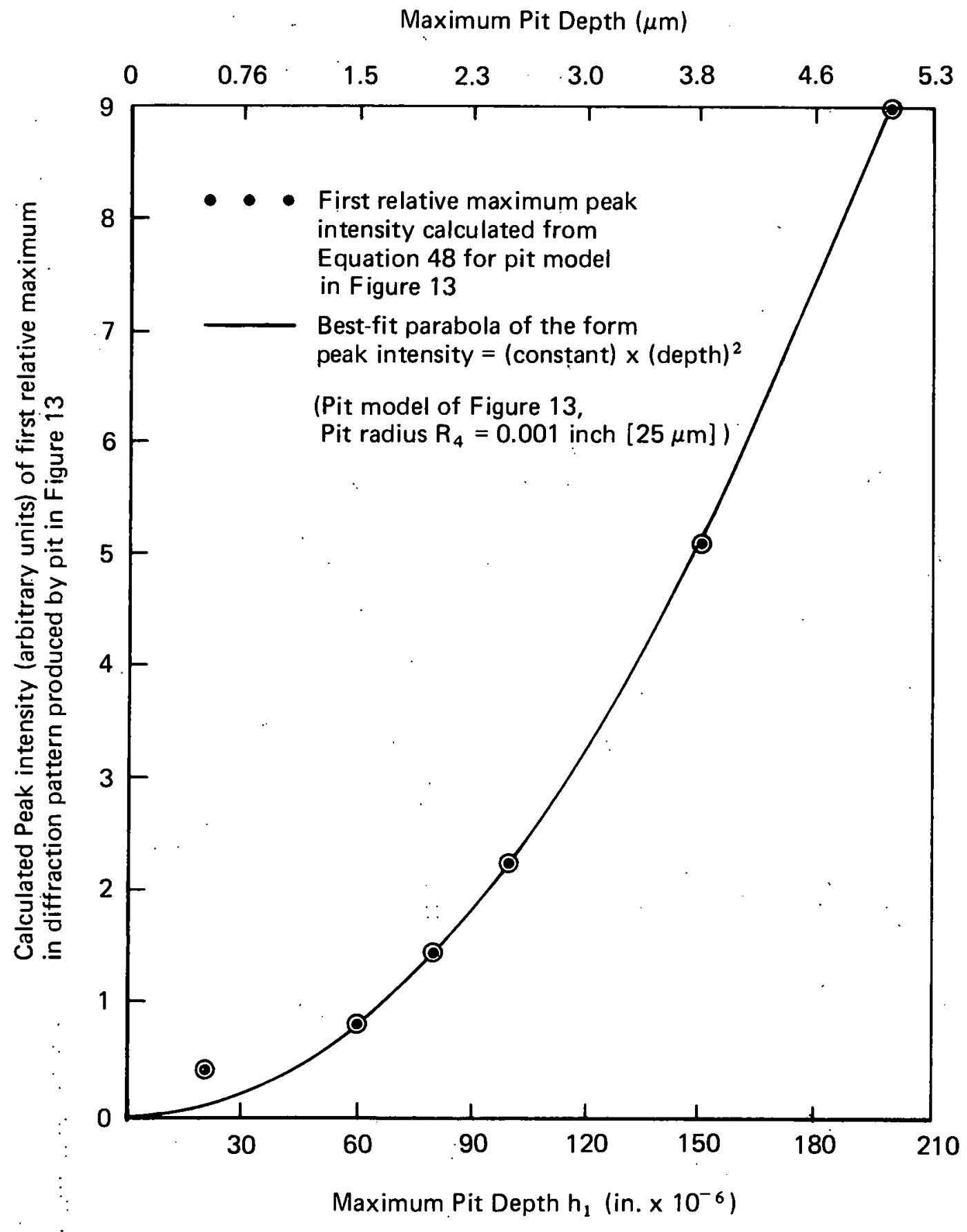

Figure 14. Calculated First Relative Maximum in Diffraction Pattern From Model Pit in Figure 13, Versus Maximum Pit Depth $h_{1}$ 


\section{FUTURE WORK}

Equipment is being procured for development of an automated surface finish inspection system for superfinished spheres. After the system is made operational, calibration data will be obtained to relate reflected light signals and diffraction pattern intensities to spherical background quality and isolated defect sizes. Further theoretical work will be done to relate diffraction pattern intensities to various detailed models of pit cross section. Experimental data will be obtained to test the theoretical dependence of diffracted intensity on defect depth for constant defect width or diameter. 


\section{REFERENCES}

${ }^{1}$ P. E. Klingsporn and W. C. Rutherford, "Investigation of Techniques for Automated Surface Inspection of Spheres" (Topical Report). BDX-613-1073, BWD-161, May, 1974.

${ }^{2}$ P. E. Klingsporn and W. C. Rutherford, "Reflected Light Intensity Distributions from Defects on Highly Reflective Spheres" (Topical Report). BDX-613-1174, November, 1974.

${ }^{3}$ P. E. Klingsporn and W. C. Rutherford, "The Angular Distribution of Diffuse Light Reflected from Isolated Defects on Superfinished Spheres" (Topical Report). BDX-613-1363, August, 1975.

${ }^{4}$ M. Born and E. Wolf, Principles of Optics. Second Edition, Pergamon Press (New York), 1964.

Miles V. Klein, Optics, John Wiley (New York), 1970.

${ }^{5}$ J. C. Slater and N. H. Frank, Electromagnetism. McGraw-Hill (New York), 1947.

${ }^{6}$ H. Davies, Proc. Inst. Elec. Engrs. 101, 209 (1959).

${ }^{7}$ J. O. Porteus, "Relation Between the Height Distribution of a Rough Surface and the Reflectance at Normal Incidence." J. Op. Soc. Amer., 53, 1389 (1963).

${ }^{8}$ J. M. Elson and R. H. Ritchie, "Diffuse Scattering and Surface-Plasmon Generation by Photons at a Rough Dielectric Surface”, Phys. Stat. Sol. B62, 461 (1974). 
Appendix A

TRANSFORMATION OF LIGHT WAVE

ELECTRIC FIELD AMPLITUDE FROM ONE

TRANSVERSE PLANE TO ANOTHER 


\section{TRANSFORMATION OF LIGHT WAVE ELECTRIC FIELD AMPLITUDE FROM ONE TRANSVERSE PLANE TO ANOTHER}

Let $P_{s}$ be a point on a coherent light source located at $x_{s}, y_{s}, z_{s}$ and $P$ be a point at distance $r$ from $P_{s}$, as shown in Figure A-1. The electric field amplitude $E_{p}$ at point $P$ is given by the FresnelKirchoff diffraction integral ${ }^{4}$ as

$E_{p}=\frac{i}{\lambda} \int_{-\infty}^{\infty} E\left(x_{s}, y_{s}, z_{s}\right) \frac{e^{-i \frac{2 \pi}{\lambda} r}}{r} d x_{s} d y_{s}$

where $E\left(x_{s}, y_{s}, z_{s}\right)$ is the electric field at the source point $P_{s}$. Consider two arbitrary points $P_{1}$ and $P_{2}$ as shown in Figure A-2 which have coordinates $x_{1}, y_{1}, z_{1}$ and $x_{2}, y_{2}, z_{1}+L$, respectively. According to Equation A-1, the electric field at point $P_{2}$, in terms of the field at point $P_{1}$, is

$E\left(x_{2}, y_{2}, z_{1}+L\right)=\frac{i}{\lambda} \int_{-\infty}^{\infty} E\left(x_{1}, y_{1}, z_{1}\right) \frac{e^{-i \frac{2 \pi}{\lambda} \sqrt{\left(x_{2}-x_{1}\right)^{2}+\left(y_{2}-y_{1}\right)^{2}+L^{2}}}}{\sqrt{\left(x_{2}-x_{1}\right)^{2}+\left(y_{2}-y_{1}\right)^{2}+L^{2}}} d x_{1} d y_{1}$.

The approximations $\mathrm{r} \approx \mathrm{L}+\frac{\left(\mathrm{x}_{2}-\mathrm{x}_{1}\right)^{2}+\left(\mathrm{y}_{2}-\mathrm{y}_{1}\right)^{2}}{2 \mathrm{~L}} \approx \mathrm{L}$ can be made when $\mathrm{L}>>\left(\mathrm{x}_{2}-\mathrm{x}_{1}\right),\left(\mathrm{y}_{2}-\mathrm{y}_{1}\right)$. Under this approximation the electric field expression in Equation A-2 can be written

$E\left(x_{2}, y_{2}, z_{1}+L\right)=\frac{i e^{-i \frac{2 \pi}{\lambda} L}}{\lambda L} \int_{-\infty}^{\infty} E\left(x_{1}, y_{1}, z_{1}\right) e^{-i \frac{2 \pi}{\lambda} \frac{\left(x_{2}-x_{1}\right)^{2}+\left(y_{2}-y_{1}\right)^{2}}{2 L}} d x_{1} d y_{1}$.

Equation A-3 is an integral transformation relating the light wave electric field amplitude in the transverse plane at $z=z_{1}+L$ to the field in the plane at $z=z_{1}$, under the condition that the distance $L$ between the transverse planes is large compared with $\left(x_{2}^{\prime}-x_{1}\right)$ and $\left(y_{2}-y_{1}\right)$. 


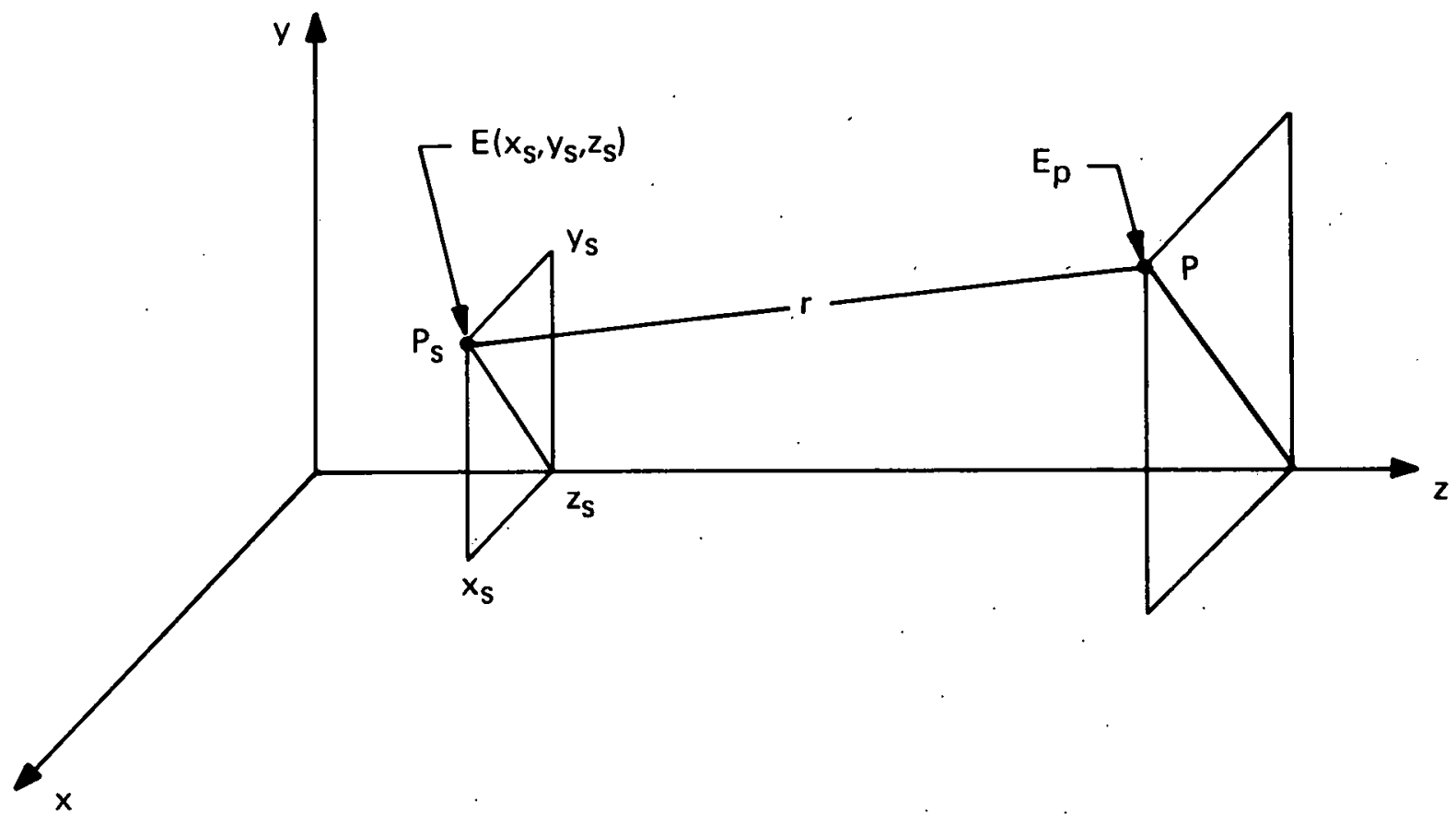

Figure A-1. Light Wave.Electric Field at a Source Point $\mathbf{P}_{\mathrm{S}}$ and at a Point $\mathbf{P}$ at Distance $r$ From $\mathbf{P}_{\mathrm{S}}$

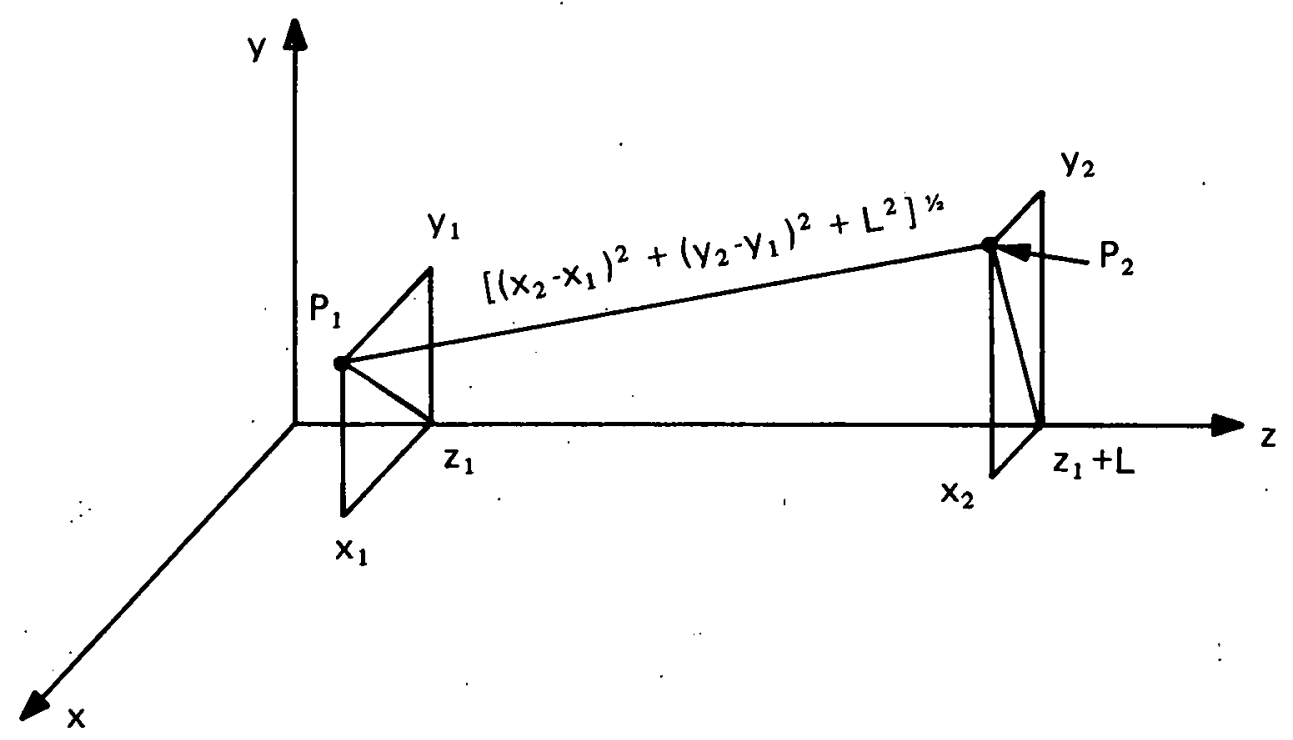

Figure A-2. Geometry Relating to the Light Wave Electric Field Transformation From Plane $\left(\mathrm{x}_{1}, \mathrm{y}_{1}, \mathrm{z}_{1}\right)$ to Plane $\left(\mathrm{x}_{2}, \mathrm{y}_{2}, \mathrm{z}_{1},+\mathrm{L}\right)$ 
Appendix B

LIGHT WAVE ELECTRIC FIELD TRANSFORMATION

FROM OBJECT PLANE TO IMAGE PLANE OF A LENS 


\section{Appendix B \\ LIGHT WAVE ELECTRIC FIELD TRANSFORMATION FROM OBJECT PLANE TO IMAGE PLANE OF A LENS}

Consider Figure B-1 in which a thin lens is located in a plane $x^{\prime} y^{\prime}$ at distance $S$ from a light source or object plane $x_{s} y_{s}$. The image formed by the lens is located in the image plane at distance $S^{\prime}$ beyond the lens. If $\mathrm{E}\left(\mathrm{x}_{\mathrm{s}}, \mathrm{y}_{\mathrm{s}},-\mathrm{S}\right)$ is the light wave electric field in the object plane, then according to Appendix $A$ the field in the plane $(x, y)$ just to the left of the lens is given by ${ }^{4}$

$E\left(x^{\prime}, y^{\prime}, 0\right)=\frac{i e^{-i k S}}{\lambda S} \int_{-\infty}^{\infty} E\left(x_{s}, y_{s},-S\right) e^{-i k \frac{\left(x^{\prime}-x_{s}\right)^{2}+\left(y^{\prime}-y_{s}\right)^{2}}{2 S}} d x_{s} d y_{s}$

Let the aperturing and focusing effects of the lens on the light passing through it be described by the transmission function

$T_{A_{\ell}}\left(x^{\prime} ; y^{\prime}\right) e^{i k \frac{x^{\prime 2}+y^{\prime 2}}{2 f}}$

where $f$ is the focal length of the lens and the factor $e^{i k \frac{x^{\prime 2}+y^{\prime 2}}{2 f}}$ represents the phase shift of the light passing through the lens. The factor $T_{A} \ell\left(x^{\prime}, y^{\prime}\right)$ is unity for an assumed infinite lens aperture. The light wave electri. .ield in the image plane to the right of the lens can be obtained from Equations B-1 and B-2 through a transformation of the type derived in Appendix A, and the result is

$E\left(x, y, S^{\prime}\right)=\frac{-e^{-i k\left(S+S^{\prime}\right)}}{\lambda^{2} S S^{\prime}} \iiint \int_{-\infty}^{\infty} E\left(x_{s}, y_{s},-S\right) T_{A} l^{\left(x^{\prime} y^{\prime}\right) e^{-i k \frac{\left(x_{s}-x^{\prime}\right)^{2}+\left(y_{s}-y^{\prime}\right)^{2}}{2 S}}}$

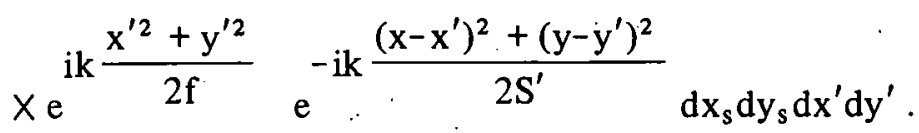

If the exponentials are expanded and like terms are collected, the phase factor in Equation B-3 can be separated into " $x$ " and " $y$ " parts as given below. 


$$
\begin{aligned}
\text { phase }= & -i \frac{k}{2}\left[x^{\prime 2}\left(\frac{1}{S}-\frac{1}{F}+\frac{1}{S^{\prime}}\right)+\frac{x_{s}^{2}}{S}+\frac{x^{2}}{S^{\prime}}-2 x^{\prime}\left(\frac{x_{s}}{S}+\frac{x}{S^{\prime}}\right)\right] \\
& -i \frac{k}{2}\left[y^{\prime 2}\left(\frac{1}{S}-\frac{1}{F}+\frac{1}{S^{\prime}}\right)+\frac{y_{s}^{2}}{S}+\frac{y^{2}}{S^{\prime}}-2 y^{\prime}\left(\frac{y_{S^{\prime}}}{S}+\frac{y}{S^{\prime}}\right)\right]
\end{aligned}
$$

The phase terms can be simplified by using the thin lens relation $1 / \mathrm{S}+1 / \mathrm{S}^{\prime}=1 / \mathrm{F}$, and therefore Equation B-3 can be written

$$
\begin{aligned}
& E\left(x, y, S^{\prime}\right)=\frac{-e^{-i k\left(S+S^{\prime}\right)}}{\lambda^{2} S S^{\prime}} \iint_{-\infty}^{\infty} E\left(x_{s}, y_{s},-S\right) e^{-i \frac{k}{2}\left[\frac{x_{s}^{2}+y_{s}^{2}}{S}+\frac{x^{2}+y^{2}}{S^{\prime}}\right]} \\
& x\left[\int_{-\infty}^{\infty} T_{A} \ell^{\left(x^{\prime}, y^{\prime}\right) e\left\{i k\left[\left(\frac{x_{s}}{S}+\frac{x}{S^{\prime}}\right) x^{\prime}+\left(\frac{y_{s}}{S}+\frac{y}{S^{\prime}}\right) y^{\prime}\right]\right\}} d x^{\prime} d y^{\prime}\right] d x_{s} d y_{s}
\end{aligned}
$$

If quantities $u$ and $v$ are defined by.

$u \equiv-\frac{1}{\lambda}\left(\frac{x_{s}}{S}+\frac{x}{S^{\prime}}\right)$ and $v \equiv-\frac{1}{\lambda}\left(\frac{y_{s}}{S}+\frac{y}{S^{\prime}}\right)$

then the double integral in the large bracket in Equation B-5 can be expressed as

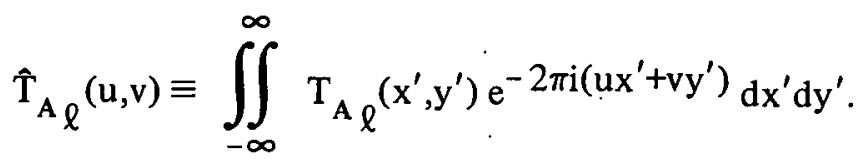

The mathematical form of Equation $B-6$ indicates that $\hat{T}_{A_{\ell}}(u, v)$ is the Fourier transform of the lens aperture transmission function $T_{A_{\ell}}\left(x^{\prime}, y^{\prime}\right)$.

If the lens aperture is assumed infinite, the four-fold integral in Equation B-5 reduces to a double integral and the resulting expression is greatly simplified. ${ }^{4}$ This can be seen by considering a rectangular aperture of dimensions $2 x_{L} \times 2 y_{L}$. The evaluation of Equation B-6 for a rectangular aperture is straightforward and leads to the following familiar result in Fraunhofer diffraction theory.

$\hat{T}_{A \ell}(u, v)=\frac{\sin \left(2 \pi u x_{L}\right)}{\pi u} \quad \frac{\sin \left(2 \pi v y_{L}\right)}{\pi v}$ 
The infinite aperture result is obtained by allowing $x_{L}$ and $y_{L}$ to approach infinity. From Dirichlet's formula,

$\operatorname{limit}_{\mathrm{L}} \rightarrow \infty \int_{-a}^{b} f\left(u+u^{\prime}\right) \frac{\sin \left(2 \pi u x_{L}\right)}{\pi u} d u=\int_{-a}^{b} f\left(u+u^{\prime}\right) \delta(u) d u=f\left(u^{\prime}\right)$

and hence each of the integrals of the form $(1 / \pi w) \sin 2 \pi w z_{\ell}$ in B-5 leads to a delta function.

Therefore, in cptical systems in which the lens apertures can be assumed infinite in relation to the dimensions in transverse planes, the "infinite lens aperture transmission function" takes the form.

$\mathrm{T}_{\mathrm{A}_{\ell I}}(\mathrm{u}, \mathrm{v}) \equiv \underset{\mathrm{y}_{\mathrm{L}} \rightarrow \infty}{\operatorname{Limit}} \underset{\mathrm{x}_{\mathrm{i}}}{\operatorname{Lim}}\left\{\mathrm{T}_{\mathrm{A}_{\ell}}(\mathrm{u}, \mathrm{v})\right\}=\delta(\mathrm{u}) \delta(\mathrm{v})$

When the change to variables $u$ and $v$ is made in Equation B-5 and Equations B-6 and B-7 are used, the electric field amplitude in the image plane can be expressed in the following form.

$E\left(x, y, S^{\prime}\right)=\frac{-e^{-i k\left(S+S^{\prime}\right)}}{\lambda^{2} S S^{\prime}} \int_{-\infty}^{\infty} E\left(x_{s}, y_{s},-S\right) e^{-\frac{i k}{2}}\left[\frac{S^{2}}{S}\left(\frac{x^{2}}{S^{\prime 2}}+u^{2} \lambda^{2}+2 u \lambda \frac{x}{S^{\prime}}\right)+\frac{S^{2}}{S}\left(\frac{y^{2}}{S^{\prime 2}}+v^{2} \lambda^{2}+2 v \lambda \frac{y}{S^{\prime}}\right)\right.$

$\left.+\frac{x^{2}+y^{2}}{S^{\prime}}\right]$

$\times \frac{\sin \left(2 \pi u x_{L}\right)}{\pi u} \frac{\sin \left(2 \pi v y_{L}\right)}{\pi v} S^{2} \lambda^{2} d u d v$.

The light wave electric field $\mathrm{E}\left(\mathrm{x}, \mathrm{y}, \mathrm{S}^{\prime}\right)$ in the infinite lens aperture approximation can be obtained from the latter expression with the aid of Equation (B-8).

$E_{A_{\ell I}}\left(x, y, S^{\prime}\right) \equiv \operatorname{Limit}_{x_{L} \rightarrow \infty}\left\{E\left(x, y, S^{\prime}\right)\right\}=\frac{-e^{-i k\left(S+S^{\prime}\right)}}{\lambda^{2} S S^{\prime}} \lambda^{2} S^{2} E\left(x_{s}, y_{s},-S\right) e^{-i \frac{k}{2}\left[S \frac{x^{2}}{S^{\prime 2}}+S \frac{y^{2}}{S^{\prime 2}}+\frac{x^{2}+y^{2}}{S^{\prime}}\right]}$

In performing the integrations in Equation B-9 to arrive at Equation B-10, the properties of the delta functions $\delta(u)$ and $\delta(v)$ were used. If the relations $x=M x_{s}, y=M y_{s}$, and $M \equiv-S^{\prime} / S$ are used, where $M$ is the magnification, then Equation B-10 can be written 
$E_{A \ell_{I}}\left(x, y, S^{\prime}\right)=\frac{1}{M} e^{-i k\left(S+S^{\prime}\right)} E\left(\frac{x}{M}, \frac{y}{M},-S\right) e^{-i \frac{k}{2}\left[\frac{x^{2}+y^{2}}{M^{2} S}+\frac{x^{2}+y^{2}}{S^{\prime}}\right]}$.

Equation B-11 can be expressed somewhat differently by referring to the geometry shown in Figure B-2. The distances $L$ and $L^{\prime}$ can be approximated by $L \approx S+x^{2} / 2 M^{2} S$ and $L^{\prime} \approx S^{\prime}+x^{2} / 2 S^{\prime}$, and hence the path aob is

$a o b \approx S+S^{\prime}+\frac{x^{2}}{2 M^{2} S}+\frac{x^{2}}{2 S^{\prime}}$

But, $\quad a o b=a c b=a c d+d b$,

and $\mathrm{db} \approx \mathrm{H}+\frac{\mathrm{x}^{2}}{2 \mathrm{H}}$

Therefore, $a o b=a c d+H+x^{2} / 2 H=a^{\prime}$ od $+H+x^{2} / 2 H=S+S^{\prime}+\frac{x^{2}}{2 H}$

because all optical paths between conjugate object and image points are the same.

The results of Equations B-12 and B-13 make it possible to put Equation B-11. in the form ${ }^{4}$

$E_{A_{\ell I}}\left(x, y, S^{\prime}\right)=\frac{1}{M} e^{-i k\left(S+S^{\prime}\right)} E\left(\frac{x}{M}, \frac{y}{M},-S\right) e^{-i \frac{k}{2}\left(\frac{x^{2}+y^{2}}{H}\right)}$.

To summarize, if the electric field amplitude in the object plane at distance $S$ to the left of a lens of focal length $F$ is given by $E\left(x_{s}, y_{s},-S\right)$, and the lens aperture is large compared with transverse dimensions of the system, then the electric field amplitude $E\left(x, y, S^{\prime}\right)$ in the image plane at distance $\mathrm{S}^{\prime}$ to the right of the lens is given by either Equation B-11 or B-14. The equations are subject to the assumption made earlier that the extents of the object and image coordinates are small compared with the object and image distances from the lens. 


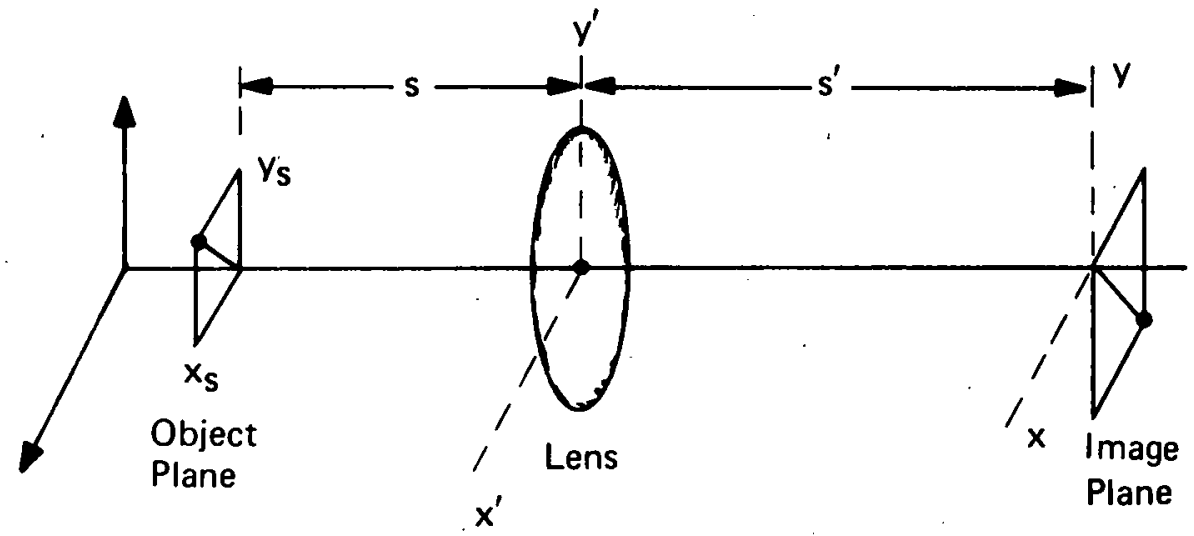

Figure B-1. Object and Image Planes for a Thin Lens

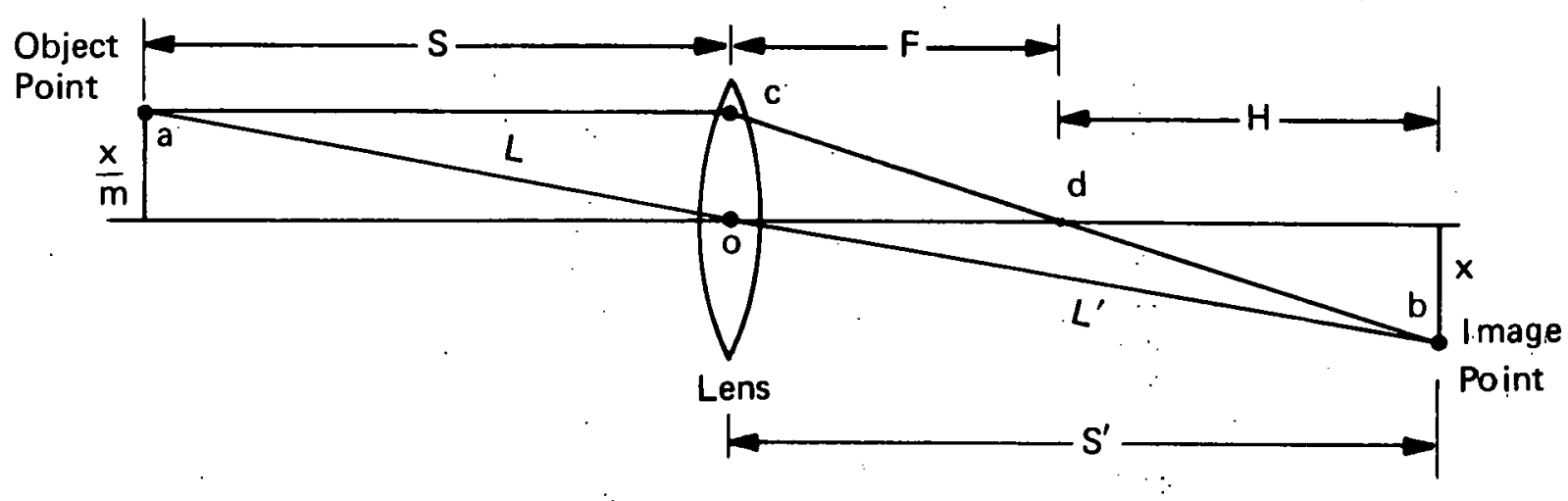

Figure B-2. Geometry Used in Obtaining for Form of Equation B-14 
Appendix C

LIGHT WAVE ELECTRIC FIELD IN THE IMAGE PLANE OF THE CENTER OF SPHERE IN TERMS OF THE

ILLUMINATED SURFACE TRANSMISSION FUNCTION 


\section{LIGHT WAVE ELECTRIC FIELD IN THE IMAGE PLANE OF THE CENTER OF SPHERE IN TERMS OF THE ILLUMINATED SURFACE TRANSMISSION FUNCTION}

According to Equation 11 of the text, a virtual point source at the center of the sphere in Figure 5 gives rise to an electric field amplitude, just to the left of the illuminated spot, given by

$E\left(x_{0}, y_{0}, S_{1}+S_{2}-R\right)=\frac{E_{0}}{R} e^{-i k R} e^{-i k \frac{x_{0}^{2}+y_{0}^{2}}{2 R}}$

where $E_{0}$ is the amplitude of the virtual point source at the sphere center and $k \equiv 2 \pi / \lambda$. After passage of the virtual light "through" the surface at the illuminated spot, the light is modulated by the illuminated surface region and hence the electric field just to the right of the spherical surface in Figure 5 is

$E\left(x, y, S_{1}+S_{2}-R\right)=\frac{E_{0}}{R} e^{-i k R} e^{-i k \frac{x^{2}+y^{2}}{2 R}} T(x, y)$

where $T(x, y)$ is the transmission function for the illuminated surface region. Equation B-14 can be used to give the light wave electric field in the image plane of the illuminated spot in Figure 5 . Let $x^{\prime}, y^{\prime}$ be the coordinates of a point in the plane in which the illuminated surface of the sphere is imaged. Then,

$$
\begin{aligned}
& E\left(x^{\prime}, y^{\prime}, S\right)=\frac{1}{M} e^{-i k\left(S_{1}+S_{2}-R+S\right)} E\left(\frac{x^{\prime}}{M}, \frac{y^{\prime}}{M}, S_{1}+S_{2}-R\right) e^{-i \frac{k}{2}\left(\frac{x^{\prime 2}+y^{\prime 2}}{S-F}\right)} \\
& =\frac{E_{0}}{M R} e^{-i k R} e^{-i k\left(S_{1}+S_{2}-R+S\right)} e^{-i k \frac{x^{\prime 2}+y^{\prime 2}}{2 M^{2} R}} \cdot T\left(\frac{x^{\prime}}{M}, \frac{y^{\prime}}{M}\right) e^{-i k \frac{x^{\prime 2}+y^{\prime 2}}{2(S-F)}} \\
& \text { or } \vdots \\
& E\left(x^{\prime}, y^{\prime}, S\right)=\frac{E_{0} e^{-i k R}}{M R} T\left(\frac{x^{\prime}}{M}, \frac{y^{\prime}}{M}\right) e^{-i k\left(S_{1}+S_{2}-R+S\right)} e^{-\frac{i k}{2}\left[\frac{x^{\prime 2}+y^{\prime 2}}{M^{2} R}+\frac{x^{\prime 2}+y^{\prime 2}}{S-F}\right]}
\end{aligned}
$$

The last exponential on the right in Equation C-3 represents the phase difference between a light ray proceeding from the sphere center to point $P_{1}$ compared with a ray passing from the center to point $P_{2}$ in Figure 5. However, all optical paths from the sphere center to the conjugate points in 
the image plane of the sphere center are the same and therefore

$$
\begin{aligned}
& -\frac{i k}{2}\left[\frac{x^{\prime 2}+y^{\prime 2}}{M^{2} R}+\frac{x^{\prime 2}+y^{\prime 2}}{S-F}\right]=-i k\left\{P_{i} P_{1}-P_{i} P_{2}\right\}=-i k\left\{P_{i} P_{1}-\left(S-S_{3}\right)\right\} \\
& \left(P_{i} P_{1}\right)^{2}=x^{\prime 2}+y^{\prime 2}+\left(S-S_{3}\right)^{2} \\
& P_{i} P_{1}=\left(S-S_{3}\right) \sqrt{1+\frac{x^{\prime 2}+y^{\prime 2}}{\left(S-S_{3}\right)^{2}}} \\
& \approx\left(S-S_{3}\right) \cdot\left[1+\frac{x^{\prime 2}+y^{\prime 2}}{2\left(S-S_{3}\right)^{2}}\right]=S-S_{3}+\frac{x^{\prime 2}+y^{\prime 2}}{2\left(S-S_{3}\right)}
\end{aligned}
$$

or

$\frac{1}{M^{2} R}+\frac{1}{S-F}=\frac{1}{S-S_{3}}$

The result in Equation C-4 allows Equation C-3 to be written

$E\left(x^{\prime}, y^{\prime}, S\right)=\frac{E_{0} e^{-i k R}}{M R} T\left(\frac{x^{\prime}}{M}, \frac{y^{\prime}}{M}\right) e^{-i k\left(S_{1}+S_{2}-R+S\right)} e^{-i k \frac{x^{\prime 2}+y^{\prime 2}}{2\left(S-S_{3}\right)}}$

This is the light wave electric field in the image plane of the illuminated surface region expressed in terms of the scaled transmission function of the illuminated spot.

When the electric field transformation equation of Appendix A is applied, the electric field in the transverse plane at $S$ given by Equation C-5 can be used to obtain the electric field in the transverse plane at $S_{3} \cdot{ }^{4}$ Let the coordinates of a point in the plane at $S_{3}$ be denoted by $(\bar{x}, \bar{y})$. The details of the transformation are given below.

$$
\begin{aligned}
& E\left(\bar{x}, \bar{y}, S_{3}\right)=\frac{i e^{-i k\left(S_{3}-S\right)}}{\lambda\left(S_{3}-S\right)} \iint_{-\infty}^{\infty} e^{-\frac{i k}{2\left(S_{3}-S\right)}\left[\left(\bar{x}-x^{\prime}\right)^{2}+\left(\bar{y}-y^{\prime}\right)^{2}\right]} E\left(x^{\prime}, y^{\prime}, S\right) d x^{\prime} d y^{\prime} \\
& =\frac{i e^{-i k\left[S_{3}+S_{1}+S_{2}\right]}}{M R \lambda\left(S_{3}-S\right)} E_{0} e^{-i k \frac{\bar{x}^{2}+\bar{y}^{2}}{2\left(S_{3}-S\right)}} \iint_{-\infty}^{\infty} T\left(\frac{x^{\prime}}{M}, \frac{y^{\prime}}{M}\right) e^{i k \frac{\bar{x} x^{\prime}+\bar{y} y^{\prime}}{\left(S_{3}-S\right)} d x^{\prime} d y^{\prime}}
\end{aligned}
$$

If the change of variables $\alpha=x^{\prime} / M$ and $\beta=y^{\prime} / M$ is made, the integral in Equation $C-6$ becomes 
$M^{2} \int_{-\infty}^{\infty} T(\alpha, \beta) e^{-2 \pi i}\left[\frac{M \bar{x}}{\lambda\left(S-S_{3}\right)} \alpha+\frac{M \bar{y}}{\lambda\left(S-S_{3}\right)} \beta\right] d \alpha \mathrm{d} \beta$

and if $u$ and $v$ are defined by $u \equiv \frac{M \bar{x}}{\lambda\left(S-S_{3}\right)}$ and $v \equiv \frac{M \bar{y}}{\lambda\left(S-S_{3}\right)}$, the result can be written

$M^{2} \int_{-\infty}^{\infty} T(\alpha, \beta) e^{-2 \pi i(u \alpha+v \beta)} \mathrm{d} \alpha \mathrm{d} \beta \equiv \mathrm{F}(\mathrm{u}, \mathrm{v})$

where $F(u, v)$ is the Fourier transform of the illuminated spot transmission function $T(\alpha, \beta)$.

Consequently, the electric field in Equation C- 6 at a point $\bar{x}, \bar{y}$ in the transverse plane at $S_{3}$ can be expressed in the following form.

$E\left(\bar{x}, \bar{y}, S_{3}\right)=\frac{-i M e^{-i k\left(S_{1}+S_{2}+S_{3}\right)}}{R \lambda\left(S-S_{3}\right)} E_{0} e^{\frac{i k\left(\bar{x}^{2}+\bar{y}^{2}\right)}{2\left(S-S_{3}\right)}} F\left[\frac{M \bar{x}}{\lambda\left(S-S_{3}\right)}, \frac{M \bar{y}}{\lambda\left(S-S_{3}\right)}\right]$ 
Appendix D

DIFFRACTED INTENSITY VERSUS

ISOLATED SCRATCH DEPTH 


\section{Appendix D}

\section{DIFFRACTED INTENSITY VERSUS ISOLATED SCRATCH DEPTH}

Derivation of Equation 36 of Text

$$
\begin{aligned}
& A=\int_{x} \int_{y} z(x) e^{-i \vec{k} \cdot \vec{r}} d x d y \\
& =-h \int_{-\frac{L}{2}}^{\frac{L}{2}} \int_{-\frac{w}{2}}^{0}\left(1+\frac{2 x}{w}\right) e^{-i\left(k_{x} x+k_{y} y\right)} d x d y-h \int_{-\frac{L}{2}}^{\frac{L}{2}} \int_{0}^{\frac{w}{2}}\left(1-\frac{2 x}{w}\right) e^{-i\left(k_{x} x+k_{y} y\right)} d x d y
\end{aligned}
$$$$
A=-\frac{2 h \sin \left(k_{y} L / 2\right)}{k_{y}}\left\{\int_{-\frac{w}{2}}^{0}\left(1+\frac{2 x}{w}\right) e^{-i k_{x} x} d x+\int_{0}^{\frac{w}{2}}\left(1-\frac{2 x}{w}\right) e^{-i k_{x} x} d x\right\}
$$

The two integrals over $\mathrm{x}$ can be combined by first changing the order of integration and substituting $\mathrm{u}=-\mathrm{x}$ in the first integral and then changing the "passive" variable $\mathrm{x}$ in the second integral to the variable $u$ as shown on the following page. 


$$
\begin{aligned}
& \int_{-\frac{w}{2}}^{0}\left(1+\frac{2 x}{w}\right) e^{-i k_{x} x} d x=-\int_{0}^{-\frac{w}{2}}\left(1+\frac{2 x}{w}\right) e^{-i k_{x} x} d x \\
& =\int_{0}^{\frac{w}{2}}\left(1-\frac{2 u}{w}\right) e^{i k_{x} u} d u
\end{aligned}
$$

Then $\int_{-\frac{w}{2}}^{0}\left(1+\frac{2 x}{w}\right) e^{-i k_{x} x} d x+\int_{0}^{\frac{w}{2}}\left(1-\frac{2 x}{w}\right) e^{-i k_{x} x} d x$

$=\left.\frac{2}{\mathrm{k}_{\mathrm{x}}} \sin \mathrm{k}_{\mathrm{x}} \mathrm{u}\right|_{0} ^{\frac{\mathrm{w}}{2}}-\left.\frac{4}{\mathrm{wk}_{\mathrm{x}}^{2}}(\cos \mathrm{v}+\mathrm{v} \sin \mathrm{v})\right|_{0} ^{\mathrm{k}_{\mathrm{x}} \frac{\mathrm{w}}{2}}$

$=\frac{4}{w_{\mathrm{x}}^{2}} \quad\left(1-\cos \mathrm{k}_{\mathrm{x}} \frac{\mathrm{w}}{2}\right)$.

Using the latter, the expression for $\mathrm{A}$ on the previous page can be written as

$$
A=4 h \frac{L \sin \left(k_{y} \frac{L}{2}\right)}{L\left(\frac{k_{y}}{2}\right)}\left[\frac{\cos \left(k_{x} \frac{w}{2}\right)-1}{w k_{x}^{2}}\right] \text {. }
$$

For scratches with length $L$ such that $k_{y} L>>1$, the factor $\left[\sin \left(k_{y} L / 2\right)\right] /\left(k_{y} L / 2\right)$ approaches the delta function $\delta\left(\mathrm{k}_{\mathrm{y}}\right)$. This means that $\mathrm{A}$ is zero except for $\mathrm{k}_{\mathrm{y}}=0$, where its value is one. The diffracted light intensity is proportional to the square of the light amplitude diffracted by the scratch:

$I \propto A^{2}=\frac{\left[\cos \left(k_{x} \frac{w}{2}\right)-1\right]^{2}}{w^{2} k_{x}^{4}}$

The quantity $\mathrm{k}_{\mathrm{x}}$ is $\mathrm{k} \sin \theta=\frac{2 \pi}{\lambda} \sin \theta$ where $\theta$ is the angle relative to the surface normal and represents the angle at which the diffracted light is observed. 
Appendix E

DIFFRACTED INTENSITY VERSUS ISOLATED PIT DEPTH FOR PIT WITH CONSTANT CONE ANGLE 


\section{Appendix E}

\section{DIFFRACTED INTENSITY VERSUS ISOLATED PIT DEPTH FOR PIT WITH CONSTANT CONE ANGLE}

Derivation of Equation 43 of Text

$A=-h \int_{r=0}^{R} \int_{\alpha=0}^{2 \pi}\left(1-\frac{r}{R}\right) r e^{-i k r s i n} \theta \cos (\alpha-\varphi) d \alpha d r$

Without loss of generality, the angle $\varphi$ can be set equal to zero., This will correspond to calculated intensity distributions in the xz plane of Figure 11. Because of symmetry, the results will be the same in any plane perpendicular to the xy plane and containing the $\mathrm{z}$ axis. Therefore,

$A=-h \int_{0}^{R} \int_{0}^{2 \pi}\left(1-\frac{r}{R}\right) r e^{-i k r \sin \theta \cos \alpha} d \alpha d r$

If the Bessel function identity $J_{0}(t)=\frac{1}{2 \pi} \int_{0}^{2 \pi} e^{i t \cos w} d w$

is used, the integration over $\alpha$ can be performed and the result is

$$
\begin{aligned}
& A=-\frac{h}{2 \pi} \int_{0}^{R}\left(1-\frac{r}{R}\right) r J_{0}(k r \sin \theta) d r \\
& =-\frac{h}{2 \pi}\left[\int_{0}^{R} r_{0}(k r \sin \theta) d r-\frac{1}{R} \int_{0}^{R} r^{2} J_{0}(k r \sin \theta) d r\right]
\end{aligned}
$$

Let $\mathrm{u} \equiv \mathrm{kr} \sin \theta$; then $\mathrm{dr}=(\mathrm{k} \sin \theta)^{-1} \mathrm{du}$. The definition of the zero order Bessel function $\mathrm{J}_{0}(\mathrm{v})$ is

$$
J_{0}(v)=\sum_{\ell=0}^{\infty} \frac{(-1)^{\ell} v^{2 \ell}}{2^{2 \ell}(\ell !)^{2}}
$$


and therefore the latter expression for $\mathrm{A}$ can be written

$A=-\frac{h}{2 \pi}\left[\frac{1}{k^{2} \sin ^{2} \theta} \int_{0}^{k R \sin \theta} u \sum_{\ell=0}^{\infty} \frac{(-1)^{\ell} u^{2 \ell}}{2^{2 \ell}(\ell !)^{2}} d u-\frac{1}{R^{3} \sin ^{3} \theta} \int_{0}^{k R \sin \theta} u^{2} \sum_{\ell=0}^{\infty} \frac{(-1)^{\ell} u^{2 \ell}}{2^{2 \ell}(\ell !)^{2}} d u\right]$.

The $\mathrm{u}$ and $\mathrm{u}^{2}$ factors multiplying the infinite series expressions in the relation for $\mathrm{A}$ above can be included under the summation signs, and term-by-term integration of the series can be performed as follows.

$$
\begin{aligned}
& A=-\frac{h}{2 \pi}\left[\frac{1}{\mathrm{k}^{2} \sin ^{2} \theta} \int_{0}^{\mathrm{kR} \sin \theta} \sum_{\ell=0}^{\infty} \frac{(-1)^{\ell} \mathrm{u}^{2 \ell+1}}{2^{2 \ell}(\ell !)^{2}} \mathrm{du}-\frac{1}{\mathrm{Rk}^{3} \sin ^{3} \theta} \int_{0}^{\mathrm{kR} \sin \theta} \sum_{\ell=0}^{\infty} \frac{(-1)^{\ell} \mathrm{u}^{2 \ell+2}}{2^{2 \ell}(\ell !)^{2}} \mathrm{du}\right] \\
& =-\frac{\mathrm{h}}{2 \pi}\left\{\frac{1}{\mathrm{k}^{2} \sin ^{2} \theta} \sum_{\ell=0}^{\infty} \frac{(-1)^{\ell}(\mathrm{kR} \sin \theta)^{2 \ell+2}}{2^{2 \ell}(\ell !)^{2}(2 \ell+2)}-\frac{1}{\mathrm{Rk}^{3} \sin ^{3} \theta} \sum_{\ell=0}^{\infty} \frac{(-1)^{\ell}(\mathrm{kR} \sin \theta)^{2 \ell+3}}{2^{2 \ell}(\ell !)^{2}(2 \ell+3)}\right\} \\
& \mathrm{A}=-\frac{\mathrm{h}}{2 \pi} \mathrm{R}^{2} \sum_{\ell=0}^{\infty} \frac{(-1)^{\ell}(\mathrm{kR} \sin \theta)^{2 \ell}}{2^{2 \ell}(\ell !)^{2}(2 \ell+2)(2 \ell+3)}
\end{aligned}
$$

In the latter, $k=2 \pi / \lambda$ where $\lambda$ is the wavelength of the light illuminating the pit. This expression for $A$ is Equation 42 of the text. 
Appendix F

DIFFRACTED INTENSITY VERSUS ISOLATED PIT DEPTH FOR PIT WITH VARIABLE CONE ANGLE 


\section{DIFFRACTED INTENSITY VERSSUS ISOLATED PIT DEPTH FOR PIT WITH VARIABLE CONE ANGLE}

Derivation of Equation 48 of Text

$A(1,2)=-\frac{h_{1} R_{2}-h_{2} R_{1}}{R_{2}-R_{1}} \int_{r=R_{1}}^{R_{2}} \int_{\alpha=0}^{2 \pi}\left(1-\frac{h_{1}-h_{2}}{h_{1} R_{2}-h_{2} R_{1}} r\right) e^{-i k r \sin \theta \cos \alpha} \operatorname{rdrd} \alpha$

When the Bessel function identity $J_{0}(t)=\frac{1}{2 \pi} \int_{0}^{2 \pi} e^{i t \cos x} d x$ is used and the definitions $\mathrm{H}_{12} \equiv\left(\mathrm{h}_{2} \mathrm{R}_{1}-\mathrm{h}_{1} \mathrm{R}_{2}\right) /\left(\mathrm{R}_{2}-\mathrm{R}_{1}\right)$ and $\mathrm{h}_{12} \equiv\left(\mathrm{h}_{1}-\mathrm{h}_{2}\right) /\left(\mathrm{h}_{1} \mathrm{R}_{2}-\mathrm{h}_{2} \mathrm{R}_{1}\right)$ are made, the expression for $A(1,2)$ becomes

$A(1,2)=H_{12} \int_{R_{1}}^{R_{2}} \int_{0}^{2 \pi} r\left(1-h_{12} r\right) e^{-i k r \sin \theta \cos \alpha} d r d \alpha$

$=\frac{\mathrm{H}_{12}}{2 \pi} \int_{\mathrm{R}_{1}}^{\mathrm{R}_{2}}\left(\mathrm{r}-\mathrm{h}_{12} \mathrm{r}^{2}\right) \mathrm{J}_{0}(\mathrm{krsin} \theta) \mathrm{dr}$

If the series expression for the zero order Bessel function is used,

$J_{0}(v)=\sum_{\ell=0}^{\infty}(-1)^{\ell} v^{2 \ell} / 2^{2 \ell}(\ell !)^{2}$

and if term by term integration of the series is carried out, the latter relation for $A(1,2)$ can be put in the following form. Let $\mathrm{u}=\mathrm{kr} \sin \theta$. Then $\mathrm{du}=\mathrm{k} \sin \theta \mathrm{dr}$, and 


$$
\begin{aligned}
& A(1,2)=\frac{\mathrm{H}_{12}}{2 \pi}\left\{\frac{1}{\mathrm{k}^{2} \sin ^{2} \theta} \int_{\mathrm{kR} \mathrm{R}_{1} \sin \theta}^{\mathrm{kR}} \sum_{\ell=0}^{\infty} \frac{(-1)^{\ell} \mathrm{u}^{2 \ell+1}}{2^{2 \ell}(\ell !)^{2}} \mathrm{du}-\frac{\mathrm{h}_{12}}{\mathrm{k}^{3} \sin ^{3} \theta} \int_{\mathrm{kR}_{1} \sin \theta}^{\mathrm{kR}_{2} \sin \theta} \sum_{\ell=0}^{\infty} \frac{(-1)^{\ell} \mathrm{u}^{2 \ell+2}}{2^{2 \ell}(\ell !)^{2}} \mathrm{du}\right\} \\
& =\frac{\mathrm{H}_{12}}{2 \pi}\left\{\left.\sum_{\ell=0}^{\infty} \frac{(-1)^{\ell} \mathrm{u}^{2 \ell+2}}{\mathrm{k}^{2} \sin ^{2} \theta 2^{2 \ell}(\ell !)^{2}(2 \ell+2)}\right|_{\mathrm{k} R_{1} \sin \theta} ^{\mathrm{kR} \sin \theta}-\left.\mathrm{h}_{12} \sum_{\ell=0}^{\infty} \frac{(-1)^{\ell \mathrm{u}^{2 \ell+3}}}{\mathrm{k}^{3} \sin ^{3} \theta 2^{2 \ell}(\ell !)^{2}(2 \ell+3)}\right|_{\mathrm{kR} \mathrm{R}_{1} \sin \theta} ^{\mathrm{kR} \sin \theta}\right\} \\
& =\frac{H_{12}}{2 \pi}\left\{\mathrm{R}_{2}^{2} \sum_{\ell=0}^{\infty} \frac{(-1)^{\ell}\left(\mathrm{kR}_{2} \sin \theta\right)^{2 \ell}}{2^{2 \ell}(\ell !)^{2}(2 \ell+2)}-\mathrm{R}_{1}^{2} \sum_{\ell=0}^{\infty} \frac{(-1)^{\ell}\left(\mathrm{kR}_{1} \sin \theta\right)^{2 \ell}}{2^{2 \ell}(\ell !)^{2}(2 \ell+2)}\right. \\
& \left.-\mathrm{R}_{2}^{3} \mathrm{~h}_{12} \sum_{\ell=0}^{\infty} \frac{(-1)^{\ell}\left(\mathrm{kR}_{2} \sin \theta\right)^{2 \ell}}{2^{2 \ell}(\ell !)^{2}(2 \ell+3)}+\mathrm{R}_{1}^{3} \mathrm{~h}_{12} \sum_{\ell=0}^{\infty} \frac{(-1)^{\ell}\left(\mathrm{kR}_{1} \sin \theta\right)^{2 \ell}}{2^{2 \ell}(\ell !)^{2}(2 \ell+3)}\right\}
\end{aligned}
$$

or,

$$
\begin{aligned}
& A(1,2)=\frac{H_{12}}{2 \pi}\left\{\sum _ { \ell = 0 } ^ { \infty } \frac { ( - 1 ) ^ { \ell } ( k \operatorname { s i n } \theta ) ^ { 2 \ell } } { 2 ^ { 2 \ell } ( \ell ! ) ^ { 2 } ( 2 \ell + 2 ) ( 2 \ell + 3 ) } \left[R_{2}^{2(\ell+1)}\left(2 \ell\left(1-R_{2} h_{12}\right)+3-2 R_{2} h_{12}\right) .\right.\right. \\
& \left.\left.+R_{1}^{2(\ell+1)}\left(2 \ell\left(R_{1} h_{12}-1\right)-3+2 R_{1} h_{12}\right)\right]\right\}
\end{aligned}
$$

The latter is Equation 47 of the text and represents the amplitude of light diffracted at angle $\theta$ relative to the normal to the mean surface level by that portion of the pit in Figure 13 which lies between the two parallel planes at distances $h_{1}$ and $h_{2}$ below the mean surface. The radius of this portion of the pit ranges from $R_{1}$ to $R_{2}$.

From the foregoing analysis, it can be seen that the light amplitude diffracted at angle $\theta$ by the portion of the pit between the planes at $h_{2}$ and $h_{3}$ can be obtained from $A(1,2)$ by replacing 1 and 2 by 2 and 3 , respectively. Likewise, the light diffracted by the uppermost portion of the pit varying in radius from $R_{3}$ to $R_{4}$ is obtained from $A(1,2)$ by replacing the subscripts 1 and 2 by 3 and 4 , respectively.

Therefore, 


$$
\begin{aligned}
& A(2,3)=\frac{H_{23}}{2 \pi}\left\{\sum _ { \ell = 0 } ^ { \infty } \frac { ( - 1 ) ^ { \ell } ( k \operatorname { s i n } \theta ) ^ { 2 \ell } } { 2 ^ { 2 \ell } ( \ell ! ) ^ { 2 } ( 2 \ell + 2 ) ( 2 \ell + 3 ) } \left[R_{3}^{2(l+1)}\left(2 \ell\left(1-R_{3} h_{23}\right)+3-2 R_{3} h_{23}\right)\right.\right. \\
& \left.\left.+R_{2}^{2(l+1)}\left(2 \ell\left(R_{2} h_{23}-1\right)-3+2 R_{2} h_{23}\right)\right]\right\}
\end{aligned}
$$

and

$A(3,4)=\frac{H_{34}}{2 \pi}\left\{\sum_{\ell=0}^{\infty} \frac{(-1)^{\ell}(k \sin \theta)^{2 \ell}}{2^{2 \ell}(\ell !)^{2}(2 \ell+2)(2 \ell+3)}\left[R_{4}^{2(l+1)}\left(2 \ell\left(1-R_{4} h_{34}\right)+3-2 R_{4} h_{34}\right)\right.\right.$

$\left.\left.+R_{3}^{2(l+1)}\left(2 \ell\left(R_{3} h_{34}-1\right)-3+2 R_{3} h_{34}\right)\right]\right\}$.

The light intensity I diffracted by the pit into the angle $\theta$ reiative to the surface normal is proportional to the total light amplitude squared and is given by

$\mathrm{I}=\frac{(2 \pi)^{4}}{\lambda^{4} \pi^{2} \mathrm{~L}_{0}^{2}}[\mathrm{~A}(1,2)+\mathrm{A}(2,3)+\mathrm{A}(3,4)]^{2}$.

The form of the expressions for $\mathrm{A}(1,2), \mathrm{A}(2,3)$, and $\mathrm{A}(3,4)$ make it easy to generalize the model of the pit referred to in Figure 13 to include $\mathrm{N}-1$ sections which are characterized by $\mathrm{N}$ different radii. The total light amplitude diffracted at angle $\theta$ off the normal by a pit of this general type is given by the expression

$$
\begin{aligned}
& A_{\text {total }}=\sum_{i=1}^{N} \frac{H_{i, i+1}}{2 \pi}\left\{\sum _ { \ell = 0 } ^ { \infty } \frac { ( - 1 ) ^ { \ell } ( k \operatorname { s i n } \theta ) ^ { 2 \ell } } { 2 ^ { 2 \ell } ( \ell ! ) ^ { 2 } ( 2 \ell + 2 ) ( 2 \ell + 3 ) } \left[R_{i+1}^{2(\ell+1)}\left(2 \ell\left(1-R_{i+1} h_{i, i+1}\right)+3-2 R_{i+1} h_{i, i+1}\right)\right.\right. \\
& \left.\left.+R_{i}^{2(\ell+1)}\left(2 \ell\left(R_{i} h_{i, i+1}-1\right)-3+2 R_{i} h_{i, i+1}\right)\right]\right\}, \\
& \text { where } H_{i, i+1}=\frac{h_{i+1} R_{i}-h_{i} R_{i+1}}{R_{i+1}-R_{i}} \text { and } h_{i, i+1}=\frac{h_{i}-h_{i+1}}{h_{i} R_{i+1}-h_{i+1} R_{i}} .
\end{aligned}
$$

For the pit model in Figure 13, the value of $\mathrm{N}$ is four, as there are $\mathrm{N}-1$ or three sections characterized by $i=1, \cdots N$ or four different values of radius. 


\section{DISTRIBUTION}

Copy

R. Bulcock, ERDA-KCAO 1

G. O. Allshouse, LASL

E. W. Colston, LASL

T. A. Sandford, LASL $\quad$. . . 4

D. J. Sandstrom, LASL

J. J. Wechsler, LASL $\quad 6$

G. A. Broadman, LLL $\quad$. 7

J. B. Bryan, LLL

L. L. Marino, LLL $\quad 9$

J. C. Oberteuffer, LLL 10

C. B. Sanford, LLL $\quad$. 11

R. W. Selden, LLL 12

E. A. Thompson, LLL $\quad 13$

R. C. Weingart, LLL $\quad 14$

$\begin{array}{ll}\text { J. J. West, LLL } & 15\end{array}$

E. G. Kristoff, D/141, 2B30 16

J. S. Carson, D/142, BQ30 $\quad 17$

W. A. Johaningsmeir, D/231, 1D40 18

J. D. Corey, D/554, BD50 . $\quad 19-20$

L. Stratton, D/554, 2C44 21-23

R. P. Frohmberg, D/800, 2A39/B. T. Lampe, D/812, 2A27 . 24

P. E. Klingsporn, D/812, 2A27 · · 25

G. W. McEachen, D/812, 2A27 $\quad$ 26-31

C. E. Bushey, D/821, 2A36

W. G. Cooper/A. G. Fransen, D/821, 2A36 33

D. P. Roberts, D/823, $1 \mathrm{~A} 27 \quad 34$

R. E. Kessler, D/865, 2C40 35 